\title{
Prêmio Nobel de 2016: Química e Física ${ }^{+*}$
}

José Maria Filardo Bassalo

Academia Paraense de Ciências

Belém - PA

Robson Fernandes de Farias ${ }^{2}$

Universidade Federal do Rio Grande do Norte

Natal - RN

\section{Resumo}

Neste artigo, trataremos dos Prêmios Nobel de 2016: Química e Física, pois eles estão relacionados com o mesmo tema: as nanoestruturas/máquinas moleculares (concepção, fabricação e explicação teórica topológica).

Palavras-chave: Prêmio Nobel de Química de 2016; Stoddart; Sauvage; Feringa; Máquinas moleculares; Prêmio Nobel de Física de 2016; Thouless; Haldane; Kosterlitz; Transições de fases topológicas.

\begin{abstract}
In this article, we will deal with the 2016 Nobel Prizes: Chemistry and Physics, since they are related to the same theme: nanostructures / molecular machines (conception, fabrication and topological theoretical explanation).
\end{abstract}

Keywords: 2016 Chemistry Nobel Prize; Stoddart; Sauvage; Feringa; Molecular machines; 2016 Physics Nobel Prize; Thouless; Haldane; Kosterlitz; Topological phase transitions.

+ The 2016 Nobel Prize: Chemistry and Physics

* Recebido: março de 2017.

Aceito: abril de 2017.

${ }^{1}$ E-mail: jmfbassalo@gmail.com

2 E-mail: robdefarias@yahoo.com.br 


\section{Introdução}

Iniciemos este artigo com o Prêmio Nobel de Química de 2016 (PNQ/2016) que foi dividido entre os químicos, o escocês Sir James Fraser Stoddart (n.1942), o francês JeanPierre Sauvage (n.1944), e o neerlandês Bernard ("Ben”) Lucas Feringa (n. 1951) - pela concepção e fabricação de máquinas moleculares -, segundo o Comitê Nobel (CN). Registre-se que (com colaboradores) Sauvage inventou o catenano (1984); Stoddart, o rotaxano (1991); e Feringa, o motor molecular (1999). Inicialmente, faremos um breve registro sobre a vida deles.

\section{Stoddart}

Stoddart nasceu em 24 de maio de 1942, em Edinburgh, Escócia. Em Edgelaw Farm ele recebeu seus primeiros estudos em uma escola, em Carrington, pequena aldeia rural de Midlothian. Depois foi para a Melville College, situada em Edinburgh e, for fim, recebeu o Bacharelado em Ciência (Química), em 1964, e o Doutorado em Química, em 1966, ambos pela University of Edinburgh (UE). Sua Tese Some Studies on Plant Gums of the Acacia Genus, teve a orientação dos químicos ingleses Sir Edmund Langley Hirst (1898-1975) e D. M. W. Anderson. Com uma National Research Council of Canada Postdoctoral Fellowship realizou, entre 1967-1969, seu Pós-Doutoramento no Department of Chemistry na Queen's University, Kingston/Ontário, Canadá, com o químico inglês John Kenyon Netherton Jones (1912-1977). Em 1970, de posse de uma Imperial Chemical Industries (ICI) Research Fellowship, Stoddart fez mais um Pós-Doutoramento, agora com o químico inglês William David Ollis (1924-1999) que era Chefe da Cadeira de Química Orgânica do Department of Chemistry/Universidade de Sheffield (USA). A partir de 1970, Stoddart foi Lecturer in Chemistry nessa Universidade, quando passou a Reader in Chemistry, em 1982, posição na qual ficou até 1991. Enquanto esteve na USA, ele foi Professor Visitante de algumas universidades como, por exemplo, no Departamento de Bioquímica da Universidade do Paraná, em 1972 e no Department of Chemistry/University of California (Los Angeles) (UCLA), em 1978 [ano em que foi eleito Chartered Chemist/Fellow of the Royal Institute of Chemistry (CChem FRIC)], com uma Science Research Council Senior Visiting Fellowship, no grupo do químico norte-americano Donald James Gram (1919-2001; PNQ, 1987). Usando as licenças sabáticas da US, entre 1978 e 1981, Stoddart trabalhou no Catalysis Group: Imperial Chemical Industries, em Runcom, na Inglaterra, que era dirigido pelo químico inglês Warren Hewertson, sob os auspícios da Science Research Council Cooperative Research Scheme. Registre-se que, em 1980, esteve na Texas A \& M University como Professor Visitante e, nesse mesmo ano, Stoddart tornou-se Doutor em Ciência (D. Sc. - Doctor of Science), pela UE, defendendo a Tese: Some Adventures in Stereochemistry, passando então a ser Fellow of the Royal Society of Chemistry (FRSC). 
Em 1990, Stoddart foi convidado para ser Professor of Organic Chemistry da University of Birmingham (UB), na Inglaterra, na qual foi Head of the School of Chemistry da mesma, entre 1993 e 1997, sendo que neste ano de 1997 e até 2002 exerceu o Honorary Professor of Chemistry/UB. Em 1997, foi para a UCLA para ocupar o Saul Winstein Professor of Chemistry, para suceder Cram que se aposentara. Em julho de 2002, passou a ser Acting CoDirector do California NanoSystems Institute (CNSI), ficando nesse cargo até 2007. Note-se que, em maio de 2003, tornou-se Fred Kavli Chair of NanoSystems Sciences/UCLA. Em 2008, criou o Mechanostereochemistry Group devido à sua nomeação para o Board of Trustees Professor in Chemistry na Northwestern University (NU), em Illinois, e nessa Universidade foi nomeado, em 2010, Director of the Center for the Chemistry of Integrated Systems (CCIS/NU).

Além do PNQ/2016 [Design and Synthesis of Molecular Machines based on the Mechanical Bond, Nobel Lecture (08/12/2016)], é interessante destacar que Stoddart recebeu mais de 100 Prêmios ("Prizes/Awards/Medals") e Honrarias ("Fellowships/Lectureships") com nosso destaque (54) para os seguintes (en.wikipedia.org/Stoddart/Stoddart_CV): 1) Hope Prize in Chemistry (University of Edinburgh/Escócia, 1964); 2) Carbohydrate Chemistry Award of the Chemical Society (American Chemical Society/USA, 1978); 3) RSC Perkin Division Career Awards (Royal Society of Chemistry/Inglaterra, 1980-1982); 4) Leverhulme Research Fellowship (The Leverhulme Trust/Inglaterra, 1986-1989); 5) Pfizer Lecturer (Ohio State University/USA, 1991); 6) Ernest Ritchie Memorial Lecturer (Sydney University Chemical Society/Austrália, 1992); 7) Walter J. Chute Lecturer (Dalhousie University/Canadá, 1992); 8) First Janssen Lecturer (Strasbourg University/França, 1993); 9) International IzattChristensen Award in Macrocyclic Chemistry (IBC Advanced Technologies, Incorporation./USA, 1993); 10) Walker Memorial Lecture (Edinburgh University/Escócia, 1994); 11) Fellow of the Royal Society (Royal Society of London/Inglaterra, 1994); 12) Chaire Bruylants Award (University of Louvain La Neuve/Bélgica, 1994); 13) Adolf Steinhofer Foundation Award (University of Kaiserslautern/Alemanha, 1995); 14) Abbott Lecturer (University of Chicago/USA, 1995); 15) Regents Endowed Memorial Lecturer (University of Texas at Austin/USA, 1996); 16) Bernard Belleau Memorial Lecturer (McGill University, Montreal/Canadá, 1997); 17) Soong Sil Lecturer (Soong Sil University/ Coréia do Sul, 1997); 18) Bio-Mega Lecturer (University of Alberta/Canadá, 1998); 19) Humboldt Fellowship (Alexander von Humboldt Foundation/Alemanha, 1998); 20) Cope Scholar Award (American Chemical Society/USA, 1999); 21) Fellowship of the German Academy of Natural Sciences, Leopoldina (Alemanha, 1999); 22) Industrial Associates Program Lecturer (Columbia University/USA, 2000); 23) Herbert Newby McCoy Award (UCLA/USA, 2001); 24) Closs Lectureship (University of Chicago/USA, 2002); 25) Lansdowne Lectureship (University of Victoria/Canadá, 2003); 26) Nagoya Gold Medal in Organic Chemistry (University of Nagoya/Japão, 2004); 27) Polymeric Materials: Science and Engineering (PMSE) Division Arthur K. Doolittle Award (American Chemical Society/USA, 2005); 28) Honorary Professorship 
(East China University of Science and Technology/China, 2005); 29) First Novartis Lectureship [Eidgenössische Technische Hochschule (ETH) Zürich/Suíça, 2005]; 30) University of Edinburgh Alumnus of the Year 2005 Award (University of Edinburgh/Escócia, 2005); 31) Fellowship of the American Association for the Advancement of Science (American Association for the Advancement of Science/USA, 2005); 32) Foreign Membership of the Royal Netherlands Academy of Arts and Sciences (Royal Netherlands Academy of Arts and Sciences/Holanda, 2006); 33) Honorary Doctor of Science Degree (University of Twente/Holanda, 2006); 34) Appointed Knight Bachelor (Her Majesty Queen Elizabeth II/Inglaterra, 2007); 35) Jabir Ibn Hyyan (Geber) Medal (Saudi Chemical Society/Arábia Saudita, 2007); 36) King Faisal International Prize in Science (King Faisal Foundation/Arábia Saudita, 2007); 37) Tetrahedron Prize for Creativity in Organic Chemistry (Elsevier Publications/Holanda, 2007); 38) Feynman Prize in Nanotechnology (Experimental) (Foresight Institute/ USA , 2007); 39) Albert Einstein World Award of Science (World Cultural Council/México, 2007); 40) Arthur C. Cope Award (American Chemical Society/USA, 2008); 41) Honorary Fellowship (Royal Society of Edinburgh/Escócia, 2008); 42) Honorary Doctor of Science Degree (University of Sheffield/USA, 2008); 43) Davy Medal (Royal Society of London/Inglaterra, 2008); 44) Donald Davis Memorial Lectureship (University of New Orleans/USA, 2009); 45) Honorary Doctor of Science Degree (Trinity College Dublin/Irlanda, 2009); 46) Royal Medal (Royal Society of Edinburgh/Escócia, 2010); 47) Undergraduate Chemistry Council Teacher of the Year (Northwestern University/USA, 2011); 48) Honorary Fellowship (Royal Society of Chemistry/Inglaterra, 2011); 49) Fellowship (American Academy of Arts and Sciences/USA, 2012); 50) Distinguished Citizen Award (Illinois Saint Andrew Society/USA, 2012); 51) IChemE North America Chemical Engineering Project Award for Innovation (Institute of Chemical Engineers/ Inglaterra, 2013); 52) Membership (National Academy of Sciences/USA, 2014); 53) Centenary Prize (Royal Society of Chemistry/USA, 2014); e 54) Haworth Memorial Lectureship (Royal Society of Chemistry/USA, 2016).

Registre-se que Stoddart orientou cerca de 400 alunos de Mestrado, Doutorado e Pesquisadores (Pós-Doutorado e Visitantes) e publicou mais de 1.000 artigos, isoladamente e em parceria, em revistas especializadas, assim como em capítulos de livros. Ele também possui (24) patentes junto com colaboradores. Ver os sites:

<en.wikipedia.org/Stoddart/Stoddart_CV/patents> (Acesso em: 15 nov. 2016).

É interessante registrar que, dentre as patentes de Stoddart, uma delas é a do rotaxano, junto com os inventores Nigel Dennis Tinker, Sayeedha Igbal e Owen Allen Matthews [Número: 6130096; Solicitada: 20 de março de 1998; Concedida: 10 de outubro de 2000; Atribuída: British Nuclear Fuels Limited (BNFL)].

Concluindo esse resumo sobre Stoddart, anote-se que ele se casou, em 1968, com a química e humanista escocesa Norma (Agnes) Scholan Stoddart (1944-2004). Anote-se, também que, em 2013, a University of Edinburgh, instituiu o The Fraser and Norma Stoddart Prize, para alunos de Doutoramento. 


\section{Sauvage}

Sauvage nasceu em Paris, em 21 de outubro de 1944. Ele obteve seu Doutorado (Doctorat d'Etat), em 20 de novembro de 1971, na Université Louis-Pasteur (Strasbourg I), na França, sob a orientação do químico francês Jean-Marie Lehn (n.1939) que, juntamente com os químicos norte-americanos Donald James Cram (1919-2001) e Charles John Pedersen (1904-1989) (nascido na Coréia do Sul), compartilhou o Prêmio Nobel de Química de 1987 (PNQ/1987), "por sintetizarem moléculas que podem se ligarem a outras moléculas", estruturas essas denominada de criptatos (criptandos) e tratadas por ele em sua Tese de Doutorado: Les Diaza-Polyoxa-Macrobicycles et Leurs Cryptates. Em seguida, realizou seu PósDoutorado com o químico inglês Malcolm L. H. Green (n.1936), na University of Oxford, na Inglaterra.

Segundo seu CV do Institut de France/Académie de Sciences (IF/AS) [wikipedia.org. (acesso em 15/11/2016)], além do PNQ/2016 [From Chemical Topology to Molecular Machines, Nobel Lecture (08/12/2016)] Sauvage recebeu os seguintes Prêmios ("Prizes/Awards/Medals"), com destaque para: 1) Bronze Medal [Centre National de la Recherche Scientifique (CNRS)/França, 1978)]; 2) French Chemical Society Award in Coordination Chemistry (French Chemical Society/França, 1979); 3) Jean-Baptiste Dumas Award (French Academy of Sciences, 1980); 4) French Chemical Society Award in Organic Chemistry (French Chemical Society/França, 1987); 5) CNRS Silver Medal (CNRS, 1988); 6) International Izatt-Christensen Award in Macrocyclic Chemistry (IBC Advanced Technologies, Incorporation./USA, 1991); 7) Prelog Gold Medal [Eidgenössische Technische Hochschule (ETH) Zürich/Suíça, 1994]; 8) World Nessim Habif Award (University of Geneva/Suíça, 1995); 9) Member (French Academy of Sciences/França, 1997); 10) Centenary Lecturer and Medal (Royal Society of Chemistry/Inglaterra, 2000); 11) Chevalier (Légion d'Honneur/França, 2000); 12) Pierre Süe Prize (French Society of Chemistry/França, 2005); 13) Catalan Sabatier Prize (Royal Spanish Society of Chemistry/Espanha, 2005); 14) Chair of the 21st Solvay Conference in Chemistry: Noncovalent Assemblies to Molecular Machines" (Brussels/Bélgica, 28 November - 1st December, 2007); 15) Robert Barns Woodward Award in Porphyrin Chemistry [Society of Porphyrins and Phthlocyanines/International Conference on Porphyrins and Phthlocyanines (ICPP), 2008]; 16) Japan Coordination Chemistry Award (Japan Coordination Chemistry/Japão, 2009); 17) Honorary Doctorate (University of Zurich/Suíça, 2010); 18) Luigi Tartufari International Prize (Accademia Nazionale dei Lincei/Itália, 2012); 19) Blaise Pascal Medal 2012 in Chemistry (European Academy of Sciences/Europa, 2012); 20) Fellow (European Academy of Sciences/Europa, 2012); e 21) Grand Prix de la Fondation de la Maison de la Chimie (Maison de la Chimie/França, 2014).

Ainda segundo seu CV(IF/AS), Sauvage recebeu as seguintes Honrarias ("Lecturers, Visiting Professor"): 1) Albright and Wilson Lecturer (University of Warwick/Inglaterra, 1988); 2) Donders Lectureship (University of Utrecht/Holanda, 1992); 3) Lecturer (University 
of Pavia/Itália, 1992); 4) Lecturer (University of Bern/Suíça, 1992); 5) Lecturer (University of Bologna/Itália, 1993); 6)

The Pfizer Lecture (University of Sheffield/Inglaterra), 1993; 7) Lecturer (University of Louvain la Neuve/Bélgica, 1994); 8) Andrews Lecturer (University of Sydney/Austrália, 1994); 9) Earl Muetterties Memorial Lecturer (University of Berkeley/USA, 1995); 10) H. H. King Lecturer (Kansas State University/USA, 1995); 11) Reilly Lecturer (Notre Dame University/USA, 1996); 12) Frontiers in Chemical Research Lecturer (Texas A \& M University/USA, 1996); 13) Morris S. Kharasch Lecturer (University of Chicago/USA, 1998); 14) Taft Lecturer (University of California, Irvine/USA, 1999); 15) Barré Lecturer (University of Montréal/Canadá, 1999); 16) Centenary Lecturer (Royal Society of Chemistry/Inglaterra, 2000); 17) Descartes Lecture (Royal Netherland Academy of Arts and Sciences/Holanda, 2000); 18) Lecturer (University of La Laguna/Espanha, 2001); 19) Lecturer (University of Santiago de Compostella/Espanha, 2001); 20) Musher Lecture (The Hebrew University of Jerusalem/Israel, 2001); 21) Les Power Lecture (James Cook University, Townsville/Austrália, 2004); 22) Steinhofer Lecture 2006 (Technical University of Kaiserslautern/Alemanha, 2007); 23) Visiting Professor (University of Zurich/Suíça, 2009); 24) Distinguished Visiting Scholar (Northwestern University/USA, 2010); 25) Lecturer (University of Hokkaido, Sapporo/Japão, 2010); 24) Lecturer (Institute for Molecular Science, Okazaki/Japão, 2010); 26) The Henlein Hall Lectures (Bowling Green State University/USA, 2012); 27) Lecturer (University of Geneva/Suíça, 2012); e 28) The R. J. P. Williams Lecture (Oxford University/Inglaterra, 2013).

Observe-se que Sauvage foi Research Fellow do CNRS (1971-1979), Director of Research do CNRS (1979-2009), University Professor da University of Strasbourg (1981-1984), e em 2009, foi indicado Professor Emeritus da University of Strasbourg e Director of Research Emeritus, do CNRS. Orientou 40 Teses de Doutorado, ministrou 561 Lectures e publicou 509 artigos, isoladamente e com colaboradores, em revistas especializadas e em capítulos de livros (sendo que em alguns destes funcionou também como editor). Para um registro completo dessa sua atividade científica, ver seu CV(IF/AS).

\section{Feringa}

Feringa nasceu no dia 18 de maio de 1951, na Municipalidade de Viagtwedde, em Barger-Compascuum, na província de Bourtange Moor, sendo esta localizada entre as províncias do nordeste da Holanda (Groningen, Friesland e Drenthe) e que fazem fronteira com a Alemanha. Seus pais católicos, Geert Feringa (1918-1993) e Lies Hake Feringa (1924-2003), eram proprietários de uma fazenda no local de seu nascimento e na qual passou boa parte de sua vida até a juventude. Em 1969, entrou no Departamento de Química Orgânica da University of Groningen (UG), na qual obteve, com distinção, seu grau de Mestre em Ciência (MSc), em 1974. Também na UG e em 1978, Feringa obteve seu Doutoramento com a tese intitulada 
Asymmetric Oxidation of Phenols. Atropisomerism and Optical Activity, sob a orientação do químico neerlandês Hans Wijnberg (Wynberg) (1922-2011).

Em sua breve biografia (en.wikipedia.org/Feringa_CV), a partir de 1978, Feringa passou a ter, paralelamente, uma carreira acadêmica e industrial. Nesta carreira, entre 1978 e 1982, foi Research Chemist de uma das maiores empresas petrolíferas do mundo, a angloholandesa Royal Dutch Shell (RDS), em seu laboratório situado em Amsterdam, pesquisando em síntese orgânica, processos de oxidação e fotoquímica. De 1982 até 1983, continuou a ser Research Chemist da RDS, agora no Shell Biosciences Centre, em Sittingbourne, Inglaterra, trabalhando com herbicidas. Em 1983, voltou para Amsterdam, para liderar o Projeto de Catálise Homogênea, ficando lá até 1984. No período de 1979-1982, iniciou sua carreira acadêmica foi Lecturer da Hogere Technische School "Amsterdam”, e continuada em 1984 quando então foi indicado como Lecturer Organic Chemistry na sua Alma Mater (UG), na qual foi apontado, em 1988, para suceder seu mestre Wijnberg, como Professor of Organic Chemistry, ocupando a Chair of Organic Chemistry. Entre 1991 e 1995, Feringa exerceu o cargo de Chairman Department Organic and Molecular Inorganic Chemistry, da UG. Em 2004, ainda na UG, foi nomeado Jacobus H. van't Hoff Distinguished Chair of Molecular Sciences.

É oportuno registrar que, além do PNQ/2016 [The Art of Building Small, from Molecular Switches to Motors, Nobel Lecture (08/12/2016)], Feringa foi condecorado com Condecorações ("Prizes/Awards/Medals/Knight/Grand Prix" (ver verbetes na Wikipedia), tais como: 1) Pino Gold Medal (Italian Chemical Society/Itália, 1997); 2) JSPS Fellowship Award and JSPS Lectureship Japan [Japan Society for the Promotion of Science (JSPS)/Japão, 1997 ou 1998); 3) Novartis Chemistry Lectureship Award (Bristol University/Inglaterra, 20002001); 4) Guthikonda Award Lecturer (Columbia University/USA, 2003); 5) Körber European Science Award (Prize) (Körber Foundation, Hamburgh/Alemanha, 2003); 6) Spinoza Award (Prize) (Netherlands Organisation for Scientific Research/Holanda, 2004); 7) Solvias Ligand Contest Award (2004) [compartilhado com o químico norte-americano John F. Hartwig (n.1964)]; 8) Prelog Gold Medal [Eidgenössische Technische Hochschule (ETH) Zürich/Suíça, 2005]; 9) James Flack Norris Award in Physical Organic Chemistry (American Chemical Society/USA, 2007); 10) Paracelsus Medal (Prize) (Swiss Chemical Society/Suíça, 2008); 11) Appointed Knight (Her Majesty Beatrix/Holanda, 2008); 12) Chirality Medal (Società Chimica Italiana/Itália, 2009); 13) Van 't Hoff Medal (University of Amsterdam/Holanda, 2011); 14) RSC Organic Stereochemistry Award (Royal Society of Chemistry/Inglaterra, 2011); 15) Humboldt Award (Alexander von Humboldt Foundation/Alemanha, 2012); 16) Grand Prix Scientifique de la Fondation Simone et Cino del Duca (Académie des Sciences de l'Institut de France/França, 2012); 17) RSC Award for Distinguished Service (Royal Society of Chemistry/Inglaterra, 2013); 18) Lilly European Distinguished Science Award (Eli Lilly and Company/USA, 2013); 19) Marie Sklodowska-Curie Medal (Polish Chemical Society/Polônia, 2013); 20) Yamada-Koga Prize (Japanese Society for Pharmaceutical Sciences/Japão, 2013); 21) Nagoya Gold Medal in Organic Chemistry (University of 
Nagoya/Japão, 2013); 22) Theodor Förster Award (German Chemical Society \& BunsenSociety for Physical Chemistry/Alemanha, 2014); 23) Yoshida Lectureship Award (International Organic Chemical Foundation/Japão, 2014); 24) Diels-Planck Award Lecture (Kiel University/Alemanha, 2015); 25) Netherlands Chemistry and Catalysis Award (Dutch Catalysis Society/Holanda, 2015); 26) Arthur C. Cope Late Career Scholars Award (American Chemical Society/USA, 2015); 27) Chemistry for the Future Solvay Prize (Her Majesty Queen Mathilde/Bélgica, 2015); e 28) August Wilhelm von Hofmann Denkmünze (Medal) (Gesellschaft Deutsche Chemische/Alemanha, 2016).

Registre-se que Feringa foi também merecedor de outras Honrarias ("Fellow/Lecturer/Visiting Professor") (en.wikipedia.org/Feringa), com destaque para: 1) Fellow of the Royal Society (Royal Society of London/Inglaterra, 1998); 2) Visiting Professor (University Leuven/Bélgica, 1999); 3) Pfizer Lecturer (Harvard University/USA, 2000); 4) Visiting Professor (University of Santiago de Compostella/Espanha, 2001); 5) W. S. Johnson Memorial Symposium Lecturer (Stanford University/USA, 2002); 6) Novartis Lecturer (Colorado State University/USA, 2002); 7) Visiting Professor (University of Potenza/Itália, 2003); 8) Marvel Lecturer/Roger Adams Labs (University Illinois/USA, 2004); 9) Dauben Distinguished Lecturer (University of California, Berkeley/USA, 2004); 10) Gassman Lecturer (University Minnesota/USA, 2005); 11) Elected Member (Royal Netherlands Academy of Sciences/Holanda, 2006); 12) Herbert C. Brown Lecturer (Purdue University/USA, 2007); 13) The A. R. Gordon Distinguished Lecturer (University Toronto/Canadá, 2007); 14) Bristol Myers Squibb BMS Lecturer (University of Michigan/USA, 2008); 15) Dauben Lecturer (University Washington, Seattle/USA, 2009); 16) ICI Distinguished Lecturer (University of Calgary/Canadá, 2009); 17) The McRae Distinguished Lectureship (Queens University, Kingston/Canadá, 2009-2010); 18) Russell Marker Lecturer in the Chemical Sciences (Pennsylvania State University/USA, 2010); 19) Elected Member (Academia Europeae/Inglaterra, 2010); 20) Andrews Lecturer (University of New South Wales/Austrália, 2011); 21) Molecular Science Forum Lecture Professorship, Institute of Chemistry (Chinese Academy of Sciences \& Chinese Chemical Society/China, 2011); 22) Boehringer Ingelheim Lecturer (University of British Columbia, Vancouver/Canadá, 2012); 23) Hassel Lecturer (University of Oslo/Noruega, 2012); 24) DTU Lecturer (Technical University Denmark/Dinamarca, 2013); 25) Bristol Myers Squibb BMS Lecturer (University of Illinois/USA, 2013); 26) The Reuben Benjamin Sandin Lectureship (University of Alberta/Canadá, 2014); 27) Jortner Lecturer (University of Tel Aviv/Israel, 2015); 28) Siegfried Hünig Vorlesung (University of Würzburg/Alemanha, 2015); e 29) Vilsmeier Lecturer (University of Regensburg/Alemanha, 2015).

Concluindo esse resumo da carreira cientifica de Feringa, anote-se que Feringa orientou mais de 100 Teses de Doutorado, publicou mais de 650 artigos, isoladamente e com colaboradores. É Membro do Corpo Editorial de 12 Revistas internacionais e é detentor de mais de 30 patentes. Por fim, Feringa ainda recebeu outras Honrarias como: 1) Presidente da 44th 
Bürgenstock Conference patrocinada pela EUCHEMS Conference on Stereochemistry, realizada na Suíça, em 2009; 2) Vice-Presidente da Koninklijke Nederlandse Akademie van Wetenschappen (KNAM) da Royal Netherlands Academy of Sciences ("Real Academia Holandesa de Ciências"), em 2011; e 3) Tetrahedron Chair da 11th Belgian Organic Synthesis Symposium (BOSS), na Bélgica, em 2012 (en.wikipedia.org/Feringa).

\section{Máquinas moleculares}

Visto um pequeno resumo das vidas científicas dos nobelistas de Química, passemos a analisar suas contribuições e que levaram à construção das máquinas moleculares. Antes, vejamos o que são esses dispositivos nanométricos. Em 29 de dezembro de 1959, por ocasião da Reunião Anual da Sociedade Americana de Física, ocorrida no California Institute of Technology (CALTECH), o físico norte-americano Richard Philips Feynman (1918-1988; PNF, 1965) ministrou uma palestra com o título There's Plenty of Room at the Bottom ("Há Abundância no Chão de Baixo"), hoje considerada a precursora da Nanotecnologia, que é um tipo de Tecnologia que envolve nanoestruturas (com dimensões nanométricas: $1 \mathrm{~nm}=10^{-9} \mathrm{~m}=10 \AA$ ) para manipular a matéria em escalas atômicas [p.ex.: o átomo de hidrogênio $(\mathrm{H})$ tem o diâmetro $\sim 0,1 \mathrm{~nm}$ ] e moleculares [p.ex.: a molécula de água $\left(\mathrm{H}_{2} \mathrm{O}\right)$ tem o diâmetro $1 \mathrm{~nm}]$.

Agora, façamos um pequeno resumo histórico dos equipamentos construídos para chegar a observar o "chão feynmaniano". Os primeiros microscópios (que observam objetos da dimensão de um micron $\left(1 \mu \mathrm{m}=10^{-6} \mathrm{~m}=1.000 \mathrm{~nm}\right)$ foram construídos na Renascença e decorrente da pesquisa da técnica de polimento de vidros. Assim, em 1590, o óptico holandês Hans Jenssen [auxiliado por seu filho Zacharias (1580-c.1638)], utilizou uma lente côncava e uma lente convexa, de pequeno poder de aumento, e inventou o Microscópio Composto (MC). Por sua vez, o físico inglês Robert Hooke (1635-1703) inventou o MC de várias lentes e, com ele, fez observações microscópicas de insetos, plumas de aves e escamas de peixes. No entanto, sua grande descoberta ocorreu quando ele examinou a cortiça. Por intermédio de cortes delgados, Hooke observou que a estrutura da cortiça era constituída de unidades ocas, retangulares e regularmente alinhadas, as quais denominou de células. Essas observações foram descritas em seu livro de nome Micrographia, publicado em 1665. Outra grande descoberta decorrente do uso do MC foi realizada pelo microscopista holandês Anton van Leeuwenhoek (1632-1723), que era um exímio construtor de lentes muito delicadas, bem finas e de pequena distância focal (algumas não chegavam a ultrapassar a cabeça de um alfinete). Assim, a partir de 1673, utilizando-se de uma montagem na qual uma única dessas lentes era utilizada para observar objetos iluminados por um espelho côncavo, isto é, o denominado microscópio simples (MS), Leeuwenhoek foi o primeiro cientista a descobrir seres vivos unicelulares, hoje chamados de protozoários (entre dezenas e centenas de $\mu \mathrm{m}$ ). Foi também, o primeiro a descrever um espermatozóide. Contudo, sua grande descoberta ocorreu em 1683, ocasião em que descreveu as primeiras bactérias $[(0,2-30) \mu \mathrm{m}=(200-3.000) \mathrm{nm}]$. 
A Microscopia Óptica teve um grande avanço quando, em 1879, o físico inglês John William Strutt, Lord Rayleigh (1842-1910; PNF, 1908) ao estudar a difração nas lentes, mostrou que o limite de aplicação de qualquer instrumento óptico (p.ex.: microscópio e telescópio) relacionava-se com o comprimento de onda $(\lambda)$ da luz utilizada. Esse limite, que caracteriza o poder de separação (resolução) de um instrumento óptico, ficou então conhecido como o Critério de Rayleigh (CR), e é dado pela expressão: $\omega=1,22 \lambda /$ d, onde $\omega$ é a separação angular (em radianos) entre dois pontos a ser separados (observados) por uma abertura circular de diâmetro d.

Ora, como a luz utilizada nos instrumentos ópticos é limitada pelo espectro eletromagnético visível [extremo vermelho $(750 \mathrm{~nm})$ até o extremo violeta $(400 \mathrm{~nm})$ ], o CR nos mostra que quanto menor for $\lambda$, melhor será a resolução dos dispositivos ópticos. Desse modo, quando o físico francês, o Príncipe Louis Victor Pierre Raymond de Broglie (1892-1987; PNF, 1927) formulou, em $1923^{3}$, a hipótese segundo a qual os elétrons apresentam um caráter dual, isto é, onda-particula ( $\lambda=\mathrm{h} / \mathrm{p}$, onde p é o momento linear do elétron e h é a constante de Planck), surgiu a possibilidade de os elétrons serem usados para observar objetos com mais detalhes, já que o $\lambda$ a eles associado é muito menor do que o da luz visível usada nos microscópios ópticos. Essa possibilidade viabilizou-se, em 1927, quando os físicos, os norteamericanos Clinton Joseph Davisson (1881-1958; PNF, 1937) e Lester Halbert Germer (18961971)4, e os ingleses Sir George Paget Thomson (1892-1975; PNF, 1937) [filho do físico inglês Sir Joseph John Thomson (1856-1940; PNF, 1906), o descobridor do elétron, em 1897] e Alexander Reid 5 observaram a difração de elétrons (lembrar que a difração é um fenômeno físico ondulatório) em monocristais de níquel (Ni) e em finas lâminas de celulóide [plástico transparente ou translúcido, altamente inflamável, feito de nitrato $\left(\mathrm{NO}_{3}\right)$ de celulose e de cânfora $\left(\mathrm{C}_{10} \mathrm{H}_{16}\right)$ ], respectivamente. Por fim, a materialização dessa possibilidade foi realizada pelo engenheiro elétrico e físico alemão Ernst August Friedrich Ruska (1906-1988; PNF, 1986) ao inventar o Microscópio Eletrônico, conforme veremos a seguir.

Ruska estudou Engenharia Eletrônica na Universidade Técnica de Munique, entre 1925-1927, e logo em 1928 transferiu-se para a Universidade Técnica de Berlim (UTB), onde, juntamente com o físico alemão Max Knoll (1897-1969), começou a investigar a Teoria de Busch. Esta havia sido desenvolvida pelo físico alemão Hans Busch (1884-1973), em 1926, ao mostrar a analogia entre o efeito de uma bobina magnética num feixe de elétrons e o de uma lente convexa em um facho de luz. Assim, Ruska e Knoll realizaram experiências com feixe de elétrons e bobinas de focagem, usando estas para formar a imagem de uma pequena abertura com uma amplificação um pouco maior do que um $(>1)$. Nessa pesquisa investigativa sobre a focagem de elétrons, Ruska percebeu que o comprimento de focagem das ondas eletrônicas poderia ser diminuído usando uma tampinha de ferro $(\mathrm{Fe})$. O trabalho deles sobre

\footnotetext{
3 Comptes Rendus de l'Académie des Sciences de Paris, v. 177, p. 507; 548; 630, 1923.

${ }^{4}$ Nature, v. 119, p. 558, 1927; Physical Review, v. 30, p. 705, 1927.

5 Journal de Physique et le Radium, v. 7, p. 327, 1927.
} 
a microscopia eletrônica, com o qual conseguiram uma amplificação de dezessete (17) vezes, foi apresentado em 14 de junho de 1931 no Colóquio Crantz, ocorrido na UTB. Agora, trabalhando isoladamente e com mais lentes, Ruska construiu, em 1933, o primeiro Microscópio Eletrônico (ME), com uma amplificação de 7.000 vezes.

O desenvolvimento do ME de Ruska levou a aumentar seu poder de resolução até 0,5 nm. Contudo, ele apresentava uma dificuldade, qual seja: a de só permitir obter imagens bidimensionais dos objetos observados. Para contornar essa dificuldade, os físicos, o alemão Gerd K. Binnig [n.1947; PNF, 1986; Kavli Prize Nanoscience (KPN), 2016] e o suíço Heinrich Rohrer (n.1933; PNF, 1986) [que trabalhavam juntos no Laboratório de Pesquisas da International Business Machines Corporation (IBM), em Rüschlikon, Suíça], começaram a projetar o Microscópio de Tunelamento de Varredura [Scanning Tunneling Microscope (STM)], usando o famoso efeito túnel (ET), que havia sido proposto, em 1928, em trabalhos independentes do físico russo-norte-americano George Antonovich Gamov (1904-1968) ${ }^{6}$, e dos físicos, o inglês Ronald Wilfrid Gurney (1898-1953) e o norte-americano Edward Uhler Condon (1902-1974) ${ }^{7}$. Esse efeito significa a capacidade de um elétron penetrar em uma barreira de potencial de altura maior do que a sua energia cinética.

No STM, um estilete com ponta (tip) de tungstênio (wolfrâmio - W), com cerca de 0,1 nm de largura, varre a superfície de dada amostra a uma distância entre 0,5-1 nm. Assim, se uma voltagem positiva é aplicada àquela ponta, elétrons da amostra examinada chegam a essa ponta pelo EF, e uma corrente elétrica pode ser detectada. Portanto, essa corrente é sensível à distância que se encontra da superfície da amostra; uma ligeira mudança nessa distância produzirá uma significativa mudança na corrente. Desse modo, se um mecanismo de realimentação mantém a corrente constante, levantando ou baixando a ponta do STM, a varredura (scanning) desse dispositivo sobre a superfície do material resultará em um mapa topográfico dela. Esse mapa permite, então, reconhecer átomos individuais superficiais. Registre-se que a invenção do STM foi anunciada em um artigo assinado por Binnig e Rohrer e, também, pelos físicos alemães Christoph H. Gerber (n.1942; KPN 2016) e Edmund Weibel ${ }^{8}$. [Gerd Binnig and Heinrich Rohrer: Scanning Tunneling Microscopy - From Birth to Adolescence (Nobel Lecture, 08 de Dezembro de 1986]. Mais tarde, em 19869, Binnig, o físico norteamericano Calvin Forrest Quate (n.1923; KPN, 2016) e Gerber inventaram o Microscópio de Força Atômica [Atomic Force Microscope (AFM)], "objetivando de medir forças menores do que $1 \mu \mathrm{N}$ entre a superfície da ponteira (tip) e a superfície da amostra", conforme registraram naquele artigo.

\footnotetext{
${ }^{6}$ Zeitschrift für Physik, v. 51, p. 204, 1928.

${ }^{7}$ Nature, v. 122, p. 439, 1928.

${ }^{8}$ Physical Review Letters, v. 49, p. 57, 1982.

${ }^{9}$ Physical Review Letters, v. 56, p. 930, 1986.
} 
A invenção desses microscópios (STM e AFM) permitiu que o mundo científico e tecnológico dispusesse de uma nova tecnologia para sondar as escalas nanométricas dos objetos. Note-se que essa tecnologia já havia recebido o nome de Nano-Tecnologia, cunhado pelo físico e engenheiro japonês Norio Tanighuci (1912-1999), por ocasião da International Conference on Production Engineering, ocorrida em Tokyo, Japão, em 1974, na qual afirmou10: "Nano-technology" mainly consists of the processing of separation, consolidation, and deformation of materials by one atom or one molecule. ("Nano-Tecnologia" consiste principalmente de processamento de separação, consolidação e deformação dos materiais por um átomo ou uma molécula). É interessante destacar que essa nova tecnologia foi iniciada graças à invenção da Molecular Beam Epitaxy (MBE), no final da década de 1960 e começo da década de 1970, pelos engenheiros eletrônicos norte-americanos John R. Arthur, Jr. (n.1931) e Alfred Yi Cho (n.1937) (de origem chinesa), trabalhando na Bell Telephone Laboratories, invenção essa que lhes permitiu desenvolver a técnica de evaporação de alto-vácuo e apresentada em $1975^{11}$.

A partir da década de 1980 foram descobertas nanoestruturas envolvendo, basicamente, folhas de carbono (C), como veremos a seguir. Antes, vejamos como se chegou a essas folhas. Teoricamente, esse novo e revolucionário material foi estudado na segunda metade da década de 1940 e na década de 1950. Com efeito, em 194712, o físico canadense Philip Richard Wallace (1915-2006) estudou a estrutura eletrônica de banda da grafita (grafite), que é um mineral alótropo do C e um bom condutor elétrico. Em 196213, o químico alemão HannsPeter Boehm (n.1928) (com a colaboração de A. Clauss, G. O. Fischer e U. Hofmann), obteve as folhas de carbono, denominando-as de grafeno (uma combinação de grafite com o sufixo eno).

Assim, naquela década de 1980, tivemos novas nano-descobertas. Com efeito, em 198414, Sauvage, Christiane O. Dietrich-Buchecker e Jean Marc Kern conseguiram ligar duas moléculas em forma de anel para formar uma cadeia, chamada catenano. Por sua vez, em 1985 (Nature 318, p. 162), os químicos, o inglês Harold Walter Kroto (n.1939; PNQ, 1996), e os norte-americanos James R. Heath, Sean C. O’Brien, Robert Floyd Curl Junior (n.1933; PNQ, 1996) e Richard Errett Smalley (n.1943; PNQ, 1996) anunciaram a descoberta de novos materiais, os fulerenos, formados por moléculas "ocas" de C e que consiste de uma superfície curva semelhante ao grafeno, mas que contém anéis pentagonais, além dos hexagonais característicos do grafeno. O exemplo mais conhecido desses materiais é o $\mathrm{C}_{60}$ que contém 60 átomos de $\mathrm{C}$ em um arranjo semelhante a uma bola de futebol [hoje considerada como

\footnotetext{
10 Proceedings, p. 18, 1974.

11 Progress in Solid State Chemistry, v. 10, p. 157, 1975.

12 Physical Review, v. 71, p. 622, 1947.

13 Zeitschrift für Anorganische und Allgemeine Chemie, v. 316, p. 119, 1962.

14 Journal of the American Chemical Society, v. 106, p. 3043, 1962.
} 
tendo a dimensão zero (0D) por ser "oca"]. Registre-se que, em 1989, os cientistas da empresa norte-americana International Business Machines (IBM) usaram um STM e manipularam 35 átomos individuais de xenônio $(\mathrm{Xe})$ e os arranjaram em cristal resfriado de níquel (Ni) e escreveram a sigla IBM. Assim, pela primeira vez, átomos foram colocados em uma superfície plana.

Desse modo, nascera a nanotecnologia, imediatamente seguida pela primeira sintetização do fulereno, em $1990^{15}$, pelos físicos, o alemão Wolfgang Kratschmer (n.1942), o grego Konstantinos Fostiropoulos (n.1930) e os norte-americanos Lowell D. Lamb (1911-2001) e Donald R. Huffman (n.1935). Destaque-se que aquela sintetização permitiu a fabricação dos nanotubos [hoje considerados como de dimensão um (1D), por ter a forma de um "fio"] e cujos primeiros exemplares foram construídos pelo físico e inventor japonês Sumio Iijima (n.1939; KPN, 2008), em 1991'16, ao observar aspectos tubulares nas imagens da fuligem de fulereno em um ME. Esses nanotubos têm a forma helicoidal, cujo diâmetro varia entre (1-3) nm e o comprimento é de $1.000 \mathrm{~nm}$ (cerca de 100.000 vezes mais fino do que um fio de cabelo). A continuidade da nanotecnologia aconteceu logo em $1991^{17}$, quando Stoddart e seus colaboradores (P. R. Ashton, M. Grognuz, A. M. Z. Slawin e D. J. Williams) desenvolveram um rotaxano: um anel molecular inserido em um eixo molecular mais fino, podendo mover-se ao longo desse eixo. Ainda em 1991, o engenheiro norte-americano Kim Eric Drexler (n.1955) tornou-se o primeiro Doutor em Nanotecnologia ao defender, no Massachusetts Institute of Technology (MIT), em Boston, Estados Unidos, sua Tese intitulada Molecular Machinery and Manufacturing With Applications to Computation, orientada pelo cientista cognitivo norteamericano Marvin Lee Minsky (1927-2016), um dos pioneiros da Inteligência Artificial.

Ressalte-se que os fulerenos e os nanotubos foram objeto de pesquisa por parte da física-química norte-americana Mildred Spievak Dresselhaus (n.1930; KPN, 2012) ao realizar uma série de artigos e livros (com importantes contribuições), desde a década de 1990 e até hoje, escritos com a colaboração de vários cientistas (principalmente com seu marido, o físico-químico norte-americano Gene Dresselhaus) do mundo inteiro, principalmente com brasileiros da Universidade Federal de Minas Gerais, como se pode ver no site: en.wikipedia.org/Mildred_Dresselhaus. Em decorrência desse seu grandioso trabalho envolvendo nanoestruturas contendo o C, ela ficou conhecida como a Rainha da Ciência do Carbono.

No final da década de 1990, em 1999 [Nature 401 (6749), p. 152], Feringa e sua equipe (Nagatoshi Koumura, Robert W. J. Zijlstra, Richard A. van Delden e Nobuyuki Harada) desenvolveram o primeiro motor molecular, ao fazerem a lâmina de um rotor molecular girar continuamente no mesmo sentido usando um feixe de luz. Cremos ser oportuno destacar

\footnotetext{
15 Nature, v. 347, p. 354. 1990.

16 Nature, v. 354, p. 56, 1991.

17 Tetrahedron Letters, v. 32, p. 6235, 1991.
} 
que, em 2011 ${ }^{18}$, Feringa e seus colaboradores (Tibor Kudernac, Noppom Ruangsupapichat, Manfred Parschau, Beatriz Maciá, Nathalie Katsonis, Syuzanna R. Harutyunyan e Kerl-Heinz Ernst) construíram um nanocarro molecular e que se moveu em uma superfície sólida por intermédio da ponta de um STM.

A previsão teórica do grafeno registrada acima deu ensejo à "corrida de ouro" em busca de seu isolamento. Uma primeira tentativa foi realizada, em $2002{ }^{19}$ pelo casal Dresselhaus ao usar a técnica da esfoliação química. Para isso, um pedaço de grafite (3D) foi primeiro intercalado de modo que planos de grafeno (2D) eram separados por camadas de átomos e moléculas. Contudo, essa técnica resultou apenas em formar um novo material tridimensional (3D), o mesmo acontecendo com outras técnicas que formavam apenas uma espécie de "lodo" grafítico. Como a técnica de esfoliação química falhou em conseguir uma superfície bidimensional da grafita, na Inglaterra, os físicos, o russo-inglês Sir Konstantin Sergeevich Novoselov (n.1974; PNF, 2010) e o russo-holandês-inglês Sir Andre Konstantinov Geim (n.1958; PIgNF, 2000; PNF, 2010) e seus colaboradores (inclusive a esposa de Geim: Irina V. Grigorieva) começaram a desenvolver uma nova técnica, relativamente simples, constituída de fitas adesivas, do tipo "lagartixa" (gecko tape). Note-se que, há muitos séculos, filósofos e cientistas tentaram entender o mecanismo de adesão, nas paredes, dos pés das lagartixas formados de pelos ceratinados. Embora um desses pelos exerça apenas uma diminuta força em torno de $10^{-7} \mathrm{~N}$ (newtons), em consequência da força de van der Waals (ver verbete nesta série), porém, milhões desses pelos produzem uma adesão da ordem $10 \mathrm{~N} / \mathrm{cm}^{2}$, suficiente para as lagartixas manterem-se e mesmo escalarem grandes edifícios. Tendo em vista esse fato, em $2003^{20}$, Geim, Grigorieva, Novoselov, S. V. Dubonos, A. A. Zhukov e S. Yu. Shapoval fabricaram microfitas biomiméticas (densos arranjos de pilares flexíveis de plástico) que faziam o papel dos pés das lagartixas. A fabricação dessas fitas adesivas levou Geim e seu grupo de pesquisa a, finalmente, isolar o grafeno. Com efeito, em 200421, Novoselov, Geim, S. V. Morozov, D. Jiang, Yuanbo Zhang, Dubonos, Grigorieva e A. A. Firsov realizaram uma experiência na qual esfoliaram a grafita(e) com um tipo de fita adesiva (durex?) e conseguiram formar flocos formados de algumas camadas da grafita(e), que então foram depositados sobre uma bolacha (wafer) de silício ( $\mathrm{Si}$ ), cuidadosamente escolhida com determinada espessura $(315 \mathrm{~nm})$ de óxido de silício $\left(\mathrm{SiO}_{2}\right)$. Ao examinarem alguns desses flocos com um $\mathrm{MC}$, observaram que os mesmos eram identificados como planos isolados de carbono e que se comportavam como um material 2D. Estava, finalmente, isolado o grafeno. Note-se que o isolamento de cristais atômicos bidimensionais foi confirmado, em $2005^{22}$, por Novoselov, D. Jiang, F. Schedin, T. J.

\footnotetext{
18 Nature, v. 479 (7372), p. 208, 2011.

19 Advances in Physics, v. 51, p. 1, 2002.

${ }^{20}$ Nature Materials, v. 2, p. 461, 2003.

21 Science, v. 306, p. 666, 2004.

22 Proceedings of the National Academy of Sciences, USA, v. 102, p. 10451, 2005.
} 
Booth, V. V. Khotkevich, Morozov e Geim e que o grafeno foi isolado, em 2006, por A. C. Ferrari, J. C. Meyer, V. Scardaci, C. Casiraghi, M. Lazzeri, F. Mauri, S. Piscanec, D. Jiang, Novoselov, S. Roth e Geim ${ }^{23}$ e A. Gupta, G. Chen, P. Joshi, S. Tadigadapa e P. C. Eklund24, usando a microscopia Raman (vide verbete nesta série) ${ }^{25}$.

É interessante concluir essa descrição histórica sobre a nanotecnologia (nanofabricação/matéria controlada), ressaltando que ela ainda está em processo laboratorial, pois ela é desenvolvida usando microscópios (ME, STM e AFM). Ela se constituirá em uma Nova Revolução Industrial que permitirá, fundamentalmente, construir a matéria de "baixo para cima", montando átomo por átomo em 3D, eliminando a necessidade de remover material indesejado, como hoje acontece, por exemplo, usando a litografia na indústria eletrônica. Além disso, ela também será bastante importante em outras indústrias, tais como: Medicina (Biologia), Computação, Engenharia dos Materiais (Impressão 3D, com a estereolitografia), Geração de Energia etc., mudando o rumo da Economia Mundial, conforme destacam os cientistas Stephen Jesse, Albina Y. Borisevich, Jason D. Fowlkes, Andrew R. Lupini, Philip D. Rack, Raymond R. Unocic, Bobby G. Sumpter, Sergei V. Kalinin, Alex Belianinov e Olga S. Ovchinnikova, no artigo Directing Matter: Toward Atomic-Scale 3D Nanofabrication [ACS (American Chemical Society) $^{26}$.

Antes de colocarmos o ponto final nessa descrição histórica da nanotecnologia, destacaremos três trabalhos relevantes sobre essa revolucionária Tecnologia. O primeiro deles trata de uma homenagem prestada a Feynman por sua previsão visionária (em 1959) dessa revolucionária tecnologia das primeiras décadas do século 20. Com efeito, uma equipe de físicos da Technische Universiteit Delft ("Universidade Técnica de Delft"), na Holanda, composta de Floris E. Kalff, M. P. Rebergen, E. Fahrenfort, J. Girovsky, R. Toskovic, J. L. Lado, F. Fernández-Rossier e A. F. Otte codificaram, em uma área de $100 \mathrm{~nm}$ de largura, uma parte do texto: A Kilobyte Rewritable Atomic Memory, publicado na revista Nature Nanotechno$\log { }^{27}$. Registre-se que 1 kilobyte $(=8.000$ bits $)$ sendo que cada bit é representado pela posição de um único átomo de cloro $(\mathrm{C} \ell)$. Sobre temas atuais da nanotecnologia, ver: Inovações Tecnológicas, 19/07/2016; 03/03/2017).

O segundo trabalho diz respeito a uma notável descoberta realizada recentemente (agosto de 2016) e que trata do seguinte. Conforme vimos neste verbete, os microscópios ópticos (MS/MC) apresentam o seguinte intervalo de limite de aplicabilidade [(200 - 3.000) nm] para a observação de bactérias. Em vista disso, pesquisas foram realizadas no sentido de obter

\footnotetext{
${ }^{23}$ Physical Review Letters, v. 97, p. 187401, 2006.

24 Nano Letters, v. 6, p. 2667, 2006.

25 Sir Andre Konstantinov Geim, Random Walk to Graphene, Nobel Lecture (08/12/2010); Sir Konstantin Sergeevich Novoselov, Graphene: Materials in the Flatland, Nobel Lecture (08/12/2010); e Andrea Latgé, O Admirável Mundo Novo do Carbono, Ciência Hoje, v. 47, p. 14, 2010.

26 Nano Letters, v. 10, p. 5600, May. 2016.

27 Letter, Jul. 2016.
} 
tipos especiais de lentes (superlentes) para melhorar esse poder de resolução daqueles microscópios, usando para isso nanogotas de diversos materiais. Contudo, os cientistas James Norman Monks, Bing Yan e Zengbo Wang [School of Electronic, Bangor University (United Kingdom)] em parceria com Nicholas Hawkins e Fritz Vollrath [Department of Zoology, University of Oxford (United Kingdom)], usaram um pedaço cilíndrico de um fio de seda (silk) de uma aranha (spider) natural (Nephila edults) como uma superlente de um MO e conseguiram um aumento adicional de 2 a 3 vezes de seu aumento natural, transformando-o em um nanoscópio. De posse desse resultado, prepararam o texto intitulado Spider Silk: Mother Nature's Bio-Superlens ${ }^{28}$.

O terceiro trabalho ${ }^{29}$, deve-se aos pesquisadores Gerald J. Brady, Austin J. Way, Nathaniel S. Safron, Harold T. Evensen, Padma Gopalan e Michael S. Arnold (Department of Materials Science and Engineering, University of Wisconsin-Madison) e Harold T. Evensen (Department of Engineering Physics, University of Wisconsin-Platteville), e no qual informaram que haviam construído o primeiro transistor orgânico, composto de nanotubos de carbono, que alcançaram 1,9 vezes a corrente dos transistores de silício (Inovações Tecnológicas, 04/10/2016).

\section{Prêmio Nobel de Física de 2016}

Vejamos agora o PNF/2016 e que foi compartilhado pelos físicos norte-americanos [radicados nos Estados Unidos da América (EUA)] David James Thouless (n.1934) (de origem escocesa), que recebeu a metade e, a outra metade foi dividida, por Frederick Duncan Michael Haldane (n.1951) (de origem inglesa) e John Michael Kosterlitz (n.1942) (de origem escocesa) - por descobertas teóricas das transições de fase topológicas e fases topológicas da matéria -, segundo o Comitê Nobel (CN). Destaque-se que esses nobelistas elaboraram seus trabalhos teóricos, principalmente, nos anos de 1970 e 1980 (como descreveremos neste artigo), em uma época em que ninguém imaginava que a Topologia pudesse ser usada como ferramenta para qualquer coisa prática. Hoje, contudo, ela é essencial para entender os sistemas físicos, com destaque para os supercondutores, os isolantes topológicos, os vidros de spin, na computação quântica e no estudo de materiais bidimensionais (Física 2D) como, por exemplo, o grafeno, que foi analisado no PNQ/2016.

Iniciemos esse relato sobre o PNF/2016 com um breve registro sobre a sua vida.

\section{Thouless}

Thouless nasceu em 21 de setembro de 1934, em Bearsden, na Escócia (sendo hoje cidadão inglês). Realizou sua formação graduada, entre 1947 e 1952, no Winchester College,

\footnotetext{
${ }^{28}$ ACS Nano Letters 6b02641, Aug. 2016.

${ }^{29}$ Advances Sciences 2, e1601240, 02/September/2016.
} 
em Winchester, capital do condado de Hampshire (Inglaterra). Recebeu o grau de Bacharel em Artes, pelo Trinity Hall, um dos Colleges da University of Cambridge (UC) [também conhecida como Cambridge University (CU)], ainda na Inglaterra, em 1955. Sob a orientação do físico germano-norte-americano Hans Albrecht Bethe (1906-2005; PNF, 1967), Thouless defendeu a Tese de Doutorado intitulada The Application of Perturbation Methods to the Theory of Nuclear Matter, em 1958, na Cornell University, nos Estados Unidos da América (EUA). Depois de realizar um Pós-Doutoramento na University of California (UC), em Berkeley, Thouless foi convidado, em 1961, para ser o primeiro Director of Studies in Physics, do Churchill College, da UC/CC, convite esse acompanhado de um Visiting Fellow ("Professor Visitante"), permanecendo nesta posição até 1965. Neste ano, ele foi trabalhar com o físico germano-inglês Sir Rudolf Ernst Peierls (1907-1995), na University of Birmingham (UB), na Inglaterra, na qual se tornou Professor de Física-Matemática, ficando na mesma até 1978, quando voltou para os EUA, para a Yale University, como Professor de Ciência Aplicada, entre 1979 e 1980 e, por fim, neste ano de 1980, foi ser Professor de Física da University of Washington (UW), em Seattle, até 2003, quando recebeu o título de Emeritus Professor of Physics. É oportuno registrar que sua esposa Margaret Elizabeth (Scrase) Thouless (com quem casou em 1958) foi Professora Associada de Pathobiologia, na UW, entre 1980 e 2004.

Além do PNF/2016 (sem Nobel Lecture), Thouless recebeu outras honrarias (fellows, medals, members, prizes e doctorates), com destaque para: 1) Maxwell Medal and Prize [Institute of Physics (IOP) (Inglaterra e Irlanda), em 1973]; 2) Fellow of the Royal Society (FRS) (Royal Society of London, em 1979); 3) Doctorate of Science [Clare Hall (College of Cambridge University), em 1985]; 4) Wolf Prize in Physics (Wolf Foundation, em 1990); 5) Dirac Medal (IOP, em 1993); 6) Member of the U.S. National Academy of Sciences, em 1995; e 7) Lars Onsager Prize (American Physical Society, em 2000).

Os trabalhos de Thouless que o levaram ao nobelato foram iniciados na UB (19651978) quando, usando métodos topológicos complexos, estudou problemas de muitoscorpos, que permitiram a compreensão dos sistemas extensos de átomos e elétrons e dos núcleons (prótons e nêutrons), os chamados sistemas bidimensionais (Física 2D). Notese que a Topologia é uma parte da Matemática e que permite estudar as propriedades de objetos que permanecem constantes mesmo se eles foram dobrados ou deformados, mas nunca rompidos. Por exemplo, quando se deforma um quadrado em um círculo ou um tubo (que é uma superfície cilíndrica) em um toroide (superfície toroidal), ambos são topologicamente iguais, pois têm apenas um buraco. Um aspecto importante a registrar é que objetos assim tratados só podem ter um número inteiro de buracos (p.ex.: 0, 1 ou 2), mas nunca 1,5 buraco.

Vejamos como aconteceram aqueles trabalhos. Na UB, Thouless teve como um de seus colaboradores realizando pós-doutoramento, o futuro nobelista Kosterlitz. Em vista dessa colaboração, em 1972, Kosterlitz e Thouless discutiram a ideia sobre um novo tipo de ordenamento de spins nos materiais ferroelétricos, denominado de ordem de longo alcance 
topológico, relacionado com a presença de vórtices. Então, juntos, demonstraram que existem estados metaestáveis correspondendo a vórtices que são estreitamente ligados em pares quando abaixo da temperatura Curie (temperatura acima da qual uma substância ferromagnética se comporta como paramagnética), enquanto acima da mesma eles se encontram livres. Nesse trabalho, eles também identificaram um novo tipo de transição (discreta) em sistemas bidimensionais [sistemas esses que foram (e são) importantes para o estudo e construção das nanomáquinas, objeto dos: PNQ/2016 e PNF/2016, como estamos mostrando neste artigo], segundo o qual defeitos topológicos representam um papel fundamental. Note-se que essa ideia de Kosterlitz-Thouless (Transição de KosterlitzThouless) também se aplica ao entendimento de filmes supercondutores e superfluidos e, também, ela é muito importante para compreender a teoria quântica de sistemas unidimensionais em temperaturas muitos baixas. Essa ideia foi por eles apresentada em dois artigos: 1) Long Range Order and Metastability in Two-Dimensional Solids and Superfluids ${ }^{30}$; e 2) Ordering, Metaestability and Phase Transitions in Two-Dimensional Systems ${ }^{31}$. É interessante destacar que, como o físico russo Vadim L'vovich Berezinskii (1935-1980) havia descoberto antes ${ }^{32}$ o papel de defeitos topológicos em transição de fase naqueles sistemas bidimensionais em baixa temperatura com uma simetria contínua; essa transição é hoje conhecida também como Transição de Berezinskii-Kosterlitz-Thouless.

Registre-se que novos trabalhos de Thouless e Kosterlitz (alguns com outros colaboradores), também na linha da Física 2D, foram publicados e ainda na década de 1970, a saber: 1) Spherical Model of a Spin Glass ${ }^{33}$, com o físico norte-americano Robert Clark Jones (19162004); 2) Infinite Range Spin Glass with M-Component Spins ${ }^{34}$, com Jones e J. R. L. de Almeida; 3) Physics in Two Dimensions, IN: D. F. Brewer (Editor), Progress in Low Temperature, Volume 7-B, p. 373 (North Holland, Amsterdam, 1978); 4) Eigenvalue Spectrum of a Large Random Matrix with Finite Mean [Journal of Physics A: Mathematical, Nuclear and General 11, p. L45 (1978)], com Jones; e 5) Stability and Susceptibility in Parisi's Solution of a Spin Glass Model ${ }^{35}$, com Almeida. É oportuno registrar que Almeida publicou o seguinte artigo e apenas com Thouless: Stability of the Sherrington-Kirkpatrick Solution of a Spin Glass Model, Journal of Physics A: Mathematical and General 11, p. 983 (1978), e que Thouless, e os físicos norte-americanos Philip Warren Anderson (n.1923; PNF, 1977) e Richard G. Palmer (n.1949) (nascido na Inglaterra) investigaram o modelo

\footnotetext{
${ }^{30}$ Journal of Physics C: Solid State Physics, v. 5, p. 124, 1972.

31 Journal of Physics C: Solid State Physics, v. 6, p. 1181, 1973. p. $610,1972$.

33 Physical Review Letters, v. 36, p. 1277, 1976; Physica, 86-88B, p. 785, 1977.

34 Journal of Physics C: Solid State Physics, v. 11, p. L871, 1978.

35 Journal of Physics C: Solid State Physics, v. 13, p. 3271, 1980.
}

32 Zhurnal Eksperimental'noi Teoretiskoi Fiziki, v. 59, p. 907, 1970/ Soviet Physics JETP, v. 32, n. 3, p. 493, 1971; Zhurnal Eksperimental’́noi Teoretiskoi Fiziki, v. 61, p. 1144, 1971/Soviet Physics JETP, 34, n. 3, 
acima referido no texto: Solution of Solvable Model of a Spin Glass, Philosophical Magazine 35, p. 593 (1977).

A Topologia também foi usada por Thouless, para entender o Efeito Hall Quântico (EHQ). Antes, vejamos o que é esse efeito e seus dois aspectos: Inteiro e Fracionário. Em outubro de 187936, o físico norte-americano Edwin Herbert Hall (1855-1938) realizou na Universidade Johns Hopkins, nos EUA, uma experiência na qual observou que quando uma longa lâmina de ouro $(\mathrm{Au})$ e percorrida longitudinalmente por uma corrente elétrica I, é colocada normalmente às linhas de força de um campo de indução magnética $\mathrm{B}$ constante, surge, entre as laterais dessa mesma lâmina, uma diferença de potencial $\mathrm{V}_{\mathrm{H}}$, dada por: $\mathrm{V}_{\mathrm{H}}=\mathrm{IR}_{\mathrm{H}}$, onde $\mathrm{R}_{\mathrm{H}}$ ficou conhecida como resistência Hall, que é diretamente proporcional a B (módulo de B): $\mathrm{R}_{\mathrm{H}}=\mathrm{B} /\left(\right.$ ne), com $n$ representando a densidade eletrônica por $\mathrm{cm}^{3}$, e $e$ é a carga elétrica do elétron. Assim, estava descoberto o que ficou conhecido como Efeito Hall Clássico (EHC).

Em 1980, quando Thouless foi para a UW, houve uma descoberta fantástica que mudou o EHC. Com efeito, naquele ano de 1980, os físicos, os alemães Klaus von Klitzing (n.1943; PNF, 1985) e Gerhard Dorda (n.1932) e o inglês Sir Michael Pepper (n.1942), publicaram um artigo ${ }^{37}$, no qual anunciaram uma descoberta sensacional: $\mathrm{R}_{\mathrm{H}}$ não variava linearmente com B, como no caso clássico. Os gráficos dessa variação lembravam uma escada, com cada degrau separado pelo valor $\mathrm{h} /\left(\mathrm{e}^{2} \mathrm{i}\right)$, onde $\mathrm{h}$ é a constante de Planck, e $\mathrm{i}=1,2,3, \ldots$, é um número quântico inteiro apropriado.

Expliquemos o significado físico do número quântico $i$. Classicamente, elétrons sob a ação de um campo magnético intenso (B) descrevem órbitas circulares ("órbitas de ciclotron") em consequência da força de Lorentz [conceituada pelo físico holandês Hendrik Antoon Lorentz (1853-1928; PNF, 1902), em 189238, por intermédio de sua célebre expressão (na linguagem atual): $\mathrm{F}_{\mathrm{L}}=\mathrm{q} \mathrm{v} \times \mathrm{B}$, onde $\mathrm{v}$ é a velocidade da carga elétrica q. Ora, quanticamente, existe somente um conjunto discreto de órbitas permitidas com energias também discretas, que caracterizam os níveis de Landau (NL) [proposto pelo físico russo Lev Davidovich Landau (1908-1968; PNF, 1962), em: Lev Landau et Evgeny Lifc(s)hitz, Mécanique Quantique:

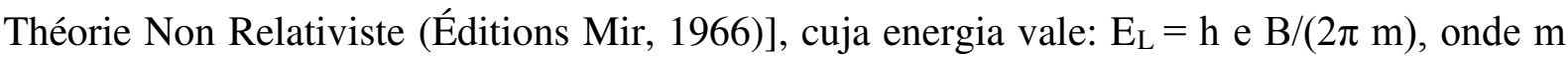
representa a massa do elétron (e). Porém, como sabemos que os elétrons com energia no interior de uma banda de energia participam da corrente de condução, a conhecida banda de condução, então, nos intervalos ("gaps") entre as bandas, os elétrons só podem ocupar os poucos níveis isolados, que são estados localizados que não participam da corrente de condução. Portanto, a ocupação desses níveis isolados não altera a resistência, resultando nos degraus observados. Desse modo, o número quântico i indica as bandas de Landau totalmente preenchidas até um dado degrau. Como o i é um número inteiro, essa descoberta de von Klitzing, Dorda e Pepper recebeu o nome de Efeito Hall Quântico Inteiro (EHQI).

\footnotetext{
36 American Journal of Mathematics, v. 2, p. 287, 1879.

37 Physical Review Letters, v. 45, p. 494, 1980.

38 Archives Néerlandaises des Sciences Exactes et Naturales, v. 25, p. 363, 1892.
} 
Por sua vez, em 198239, os físicos norte-americanos Horst Ludwig Störmer (n.1949; PNF, 1998) (de origem alemã), Daniel Chee Tsui (n.1939; PNF, 1998) (de origem chinesa) e Arthur Charles Gossard (n.1935) descobriram que: $R_{H}=\varphi_{0} /\left[(1 / 3)\right.$ i e], sendo $\varphi_{0}=h / e$, o fluxo quântico magnético. Essa nova descoberta recebeu o nome de Efeito Hall Quântico Fracionário (EHQF). Observe-se que esses efeitos (EHQ:I/F) foram explicados pelo físico norteamericano Robert B. Laughlin (n.1950; PNF, 1998), como veremos a seguir.

Em 1979, Laughlin defendeu sua Tese de Doutoramento no MIT com o físico norteamericano John D. Joannoupoulos (n.1947), que liderava o grupo de pesquisa em Física do Estado Sólido (hoje: Física da Matéria Condensada. No ano seguinte, em 1980 (o ano da descoberta do EHQI), ele trabalhava na Bell Laboratories, em Murray Hill/New Jersey. Em certo dia daquele ano, na sala de chá da Bell, Tsui conversava com um pequeno grupo de teóricos desse Laboratório, no qual se encontravam os físicos norte-americanos Philip Warren Anderson (n.1923; PNF, 1977) e Laughlin, e, com o trabalho daqueles físicos na mão, perguntou como aquele efeito poderia ser explicado. Sua dúvida decorria do fato de que os trabalhos teóricos até então realizados sobre a localização na referida Física 2D não o conseguiam explicar. Registre-se que o conceito de localização (estado isolante quando um metal nãointeragente é submetido a um grande potencial randômico) havia sido formulado por Anderson, em $1958^{40}$. Depois de ouvir Tsui, Anderson sugeriu que, para explicar o EHQI, era necessário considerar a transformação de calibre ("gauge") do potencial vetor $\mathrm{A}: \mathrm{A}$ ' $=\mathrm{A}+\mathrm{A}_{0}$, com $\mathrm{A}_{0}$ considerado como um vetor constante e sem significado físico. Para dar um significado físico a esse vetor, Laughlin imaginou uma "experiência de pensamento", na qual a fita metálica característica do EHC é enrolada em forma de anel ("loop"). Com isso, Laughlin demonstrou, em $1981^{41}$, que o módulo de $\mathrm{A}_{0}\left(\mathrm{~A}_{0}\right)$ relaciona-se com o fluxo $\varphi_{0}$ que passa pelo anel. Desse modo, considerando que esse fluxo, que é adiabaticamente forçado através do anel, é o que leva o elétron do Nível de Fermi (NF) de uma extremidade para a outra da fita. Laughlin encontrou o valor de $\mathrm{R}_{\mathrm{H}}$ do EHQI obtido por von Klitzing, Dorda e Pepper, dividindo esse fluxo pelo produto da carga elétrica do elétron (e) pelo número de ocupação (i) do NF. Note-se que a energia do NF é dada por: $\mathrm{E}_{\mathrm{F}}=-\alpha \mathrm{k}$ T onde $\alpha$ é um parâmetro e que depende da temperatura absoluta $\mathrm{T}$, do número de moléculas e da distribuição dos possíveis níveis de energia, e k é a constante de Boltzmann. Essa $E_{F}$ significa a energia mais alta em que, no zero absoluto $(\mathrm{T}=0)$, o elétron pode ocupar em sua distribuição energética. A superfície para a qual $E_{F}$ é constante chama-se superfície de Fermi. É oportuno ainda registrar que $E_{F}$ nos con-

\footnotetext{
${ }^{39}$ Physical Review Letters, v. 48, p. 1559, 1982.

40 Physical Review, v. 109, p. 1492; 112, p. 1900, 1958.

41 Physical Review B23, p. 5632, 1981.
} 
dutores situa-se na banda de condução; nos isolantes, na banda de valência; e nos semicondutores, no intervalo ("gap") entre essas duas bandas ${ }^{42}$.

Agora, vejamos a explicação do EHQF apresentada por Laughlin. Ainda em 1981, ele foi trabalhar no Lawrence Livermore National Laboratory, em Livermore, na Califórnia. Nesse Laboratório, ele vinculou-se ao grupo de plasma que tinha como principais teóricos os físicos norte-americanos Hugh Edgar DeWitt (1930-2014) e Forest Rogers. Enquanto realizava seu trabalho de pesquisa no anexo daquele Laboratório conhecido como "The Cooler", sobre o modelamento da matéria em energias da ordem de $10 \mathrm{eV}$, Laughlin recebeu o "preprint" do artigo de Tsui, Störmer e Gossard sobre a descoberta do EHQF. Essa descoberta apresentava um resultado surpreendente, isto é, a $R_{H}$ por eles observada significava três vezes a mesma resistência do EHQI. Depois de ler o artigo, Laughlin telefonou a Störmer e, ao perguntar-lhe como explicar esse surpreendente resultado, recebeu então a informação de que Tsui, inicialmente, pensara tratar-se de quarks [conceito introduzido, independentemente, em 1964, pelos físicos, o norte-americano Murray Gell-Mann (n.1929; PNF, 1969) ${ }^{43}$ e o russonorte-americano George Zweig (n.1937) (CERN Preprint, 8182/Th 401; 8419/Th 412)], pois o valor de $\mathrm{R}_{\mathrm{H}}$ encontrado correspondia a uma partícula com a carga elétrica de e/3. Contudo, Störmer disse-lhe em seguida, que o próprio Tsui logo viu a impossibilidade dessa hipótese, uma vez que a experiência que realizaram tratavam com energia da ordem de mili-elétronvolt (1 $\left.\mathrm{meV}=10^{-3} \mathrm{eV}\right)$, insuficiente para produzir quarks, que é da ordem de mega-volts $\left(1 \mathrm{MeV}=10^{6} \mathrm{eV}\right)$.

Conhecedor dessa informação, Laughlin procurou outra explicação para o EHQF. Conversando com seu estudante Eugene L. Mele sobre o conceito de quase-partícula, usado em Física de Partículas Elementares e em Física do Estado Sólido, Laughlin pensou em explicar aquele efeito como sendo devido a um condensado de muitos-corpos com excitações correspondentes a uma quase-partícula de carga e/3. Com essa ideia em mente, preparou um artigo e o enviou para a Physical Review Letters (PRL). Nesse artigo, ele mostrou que a quasepartícula observada no EHQF decorria de uma quebra de simetria discreta. No entanto, um dos "referees" [o físico norte-americano Steve Allan Kivelson (n.1954), conforme Laughlin veio a saber, ao ser anunciado que havia compartilhado o PNF de 1998] da PRL rejeitou-o, afirmando que a quebra de simetria considerada por Laughlin não era discreta e sim contínua e, portanto, sua ligação com impurezas poderia tornar isolante o material utilizado por Tsui, Störmer e Gossard. Apesar de já saber que isso era verdade enquanto preparava o artigo, Laughlin decidiu, assim mesmo, enviá-lo para a PRL, pois achava ser irrelevante esse fato. Essa rejeição foi providencial, pois lhe ajudou a encontrar a explicação correta para o EHQF, conforme veremos mais adiante. Antes, façamos alguns comentários sobre o conceito de qua-

42 KITTEL, C. Introduction to Solid State Physics. John Wiley and Sons, 1971; ZIMAN, J. M. Principles of the Theory of Solids (Cambridge University Press, 1972); CERQUEIRA LEITE, R.C.; CASTRO, A. R. B. Física do Estado Sólido. Editora Edgard Blücher Ltda., 1978.

43 Physics Letters, v. 8, p. 214, 1964. 
se-partícula. Note-se que este conceito foi proposto, em $1976^{44}$, pelos físicos, o polaco-norteamericano Roman Wladimir Jackiw (n.1939) e o italiano Claudio Rebbi (n.1943), em um trabalho em que apresentaram a ideia de que existem partículas que podem carregar parte de um número quântico elementar. Essa quase-partícula é também conhecida como sóliton topológico ou partícula kink.

Voltemos ao trabalho teórico de Laughlin sobre o EHQF. Em $1983^{45}$, ele explicou esse efeito ainda usando a ideia de quase-partícula, porém, com outra interpretação. Vejamos qual. Inicialmente, Laughlin mostrou que um gás de elétrons, sob um campo magnético intenso $[\sim 15 \mathrm{~T}$ (Tesla)] e temperatura baixa $(\sim 0.48 \mathrm{~K})$ (dados da experiência de Tsui, Störmer e Gossard), pode se condensar para formar um fluido quântico similar ao que ocorre com o hélio líquido e os supercondutores. No entanto, para explicar as cargas fracionárias características do EHQF, Laughlin imaginou uma experiência de pensamento, análoga à que considerou na explicação do EHQI, envolvendo o fluxo quântico magnético $\left(\varphi_{0}=\mathrm{h} / \mathrm{e}\right)$. Como os elétrons sofrem repulsão coulombiana, considerou que o condensado envolvia estruturas compostas de elétrons e de pequenos vórtices, um para cada fluxo quântico magnético. Desse modo, concluiu que o fluido quântico proposto comportava-se como um "superfluido" e que, quando excitado, por intermédio de mais elétrons e vórtices ("quantizados"), reage formando novos estados coletivos (quase-partículas ou excitações coletivas), com carga elétrica efetiva "fracionária". Ora, como para criar esses estados é necessário um consumo de energia, o "superfluido" resiste à compressão e, portanto, torna-se "incompressível". Assim, segundo Laughlin, o comportamento quântico dessas quase-partículas é descrito por um Hamiltoniano [Hamiltoniano de Laughlin (HL)] aplicado a seguinte função de onda de Laughlin:

$$
\psi_{\mathrm{m}}\left(\mathrm{z}_{1}, \mathrm{z}_{2}, \ldots, \mathrm{z}_{\mathrm{N}}\right)=\prod_{\mathrm{j}<\mathrm{k}}^{\mathrm{N}}\left(\mathrm{z}_{\mathrm{j}}-\mathrm{z}_{\mathrm{k}}\right)^{\mathrm{m}} \exp \left\{\left[-1 /\left(4 \ell^{2}\right)\right] \sum_{\mathrm{j}}^{\mathrm{N}}\left|\mathrm{z}_{\mathrm{j}}\right|^{2}\right\},
$$

no qual m é um inteiro ímpar (igual a 3, no caso da experiência inicial de Tsui, Störmer e Gossard), que significa o número de vórtices ("quantizados") do fluxo $\varphi_{0}, \mathrm{z}_{\mathrm{j}}=\mathrm{x}_{\mathrm{j}}+\mathrm{i} \mathrm{y}_{\mathrm{j}}$, representa a posição do j-ésimo elétron, e $(\mathrm{m})^{1 / 2} \ell$ é o comprimento magnético. É interessante notar que a ideia de essa função de onda representar um fluido quântico, em vez de um estado cristalino, foi desenvolvida por Laughlin depois de consultar livros de Mecânica Estatística e de discutir com DeWitt e Rogers, em Livermore.

Por fim, vamos concluir a contribuição de Thouless para o PNF/2016, destacando alguns de seus artigos relacionados com a explicação topológica do EHQ, na década de 1980, na UW, trabalhando com físicos que faziam o doutoramento e pós-doutoramento com ele. Logo em 198146, Thouless usou o conceito de localização para explicar o EHQ bidimensional,

\footnotetext{
44 Physical Review D13, p. 3398, 1976.

45 Anomalous Quantum Hall Effect: An Incompressible Quantum Fluid with Fractionally Charged Excitations, Physical Review Letters, v. 50, p. 1395, 1983.
}

46 Journal of Physics C: Solid State Physics, v. 14, p. 3475, 1981. 
no artigo: Localization and the Two-Dimensional Hall Effect. Em 198247, Thouless e Mahito Kohmoto, Peter M. Nightingale e Marcel den Nijs publicaram o texto: Quantized Hall Conductance in a Two-Dimensional Periodic Potencial, no qual a Condutância Quântica Hall $\left(\mathrm{C}_{\mathrm{H}}=1 / \mathrm{R}_{\mathrm{H}}\right)$ de um gás eletrônico bidimensional é estudada em um campo magnético uniforme sob um potencial periódico U. Neste artigo, a Fórmula de Kubo (FK) [deduzida pelo físico japonês Ryogo Kubo (1920-1995), em 195748, e que expressa a resposta linear de uma quantidade observável devido a uma perturbação temporal] é deduzida de modo que a quantização aparece quando os NF (Nível de Fermi) estão em um intervalo ("gap"). Por esta razão, a FK é hoje conhecida como a Fórmula de TKNN (FTKNN). Logo em 1983, por ocasião da 5th International Conference on Electronic Properties of Two-Dimensional Systems, realizada em Oxford, na Inglaterra, Thouless discutiu o problema da quantização de sistemas de muitoscorpos e bidimensionais ${ }^{49}$. Dois anos depois, em $1985^{50}$, Thouless e mais dois físicos de origem chinesa, Qian Niu e Yong-Shi Wu, generalizaram o artigo que Thouless havia escrito com Kohmoto, Nightingale e den Nijs e, ao aplicarem o EHQF observaram que deveria haver uma quebra de simetria no estado fundamental de um sistema de muitos-corpos. Com isso, eles demonstraram a possibilidade de a Condutância Quântica Hall Fracionária ser um invariante topológico. Em 198751, Thouless e Niu examinaram trabalhos anteriores sobre a dependência do EHQ das condições de fronteira ("boundary conditions") (p. ex.: cilíndrica e toroidal) e, comparando-as com as experiências reais [principalmente as que exibiam quantização (representada por riscas)], verificaram que havia uma discrepância entre esses resultados. Então, usando o conceito de quase-partícula de Laughlin (para explicar o EFHQF) e o trabalho de Thouless, Niu e Wu, os dois (Niu e Thouless) concluíram que a correção de fronteira para a $\mathrm{C}_{\mathrm{H}}$ é exponencialmente pequena quando o tamanho do sistema físico considerado é grande comparado com o comprimento microscópico magnético.

Ainda é oportuno salientar que Thouless registrou o papel da Topologia na Física Não-Relativística, no livro intitulado: Topological Quantum Numbers in Nonrelativistic Physics (World Scientific Publishing Company, 1998).

\section{Haldane}

Passemos, agora, a considerar um breve registro da vida de Haldane. Ele nasceu em 14 de setembro de 1951, em Londres e realizou seu Ensino Médio na Saint Paul's School, em Londres. Começou sua Graduação em Natural Sciences (“Ciências Naturais”), em 1970, no

\footnotetext{
${ }^{47}$ Physical Review Letters, v. 49, p. 405, 1982.

48 Journal of the Physical Society of Japan, v. 12, p. 570, 1957.

49 É importante anotar que Thouless havia discutido esses sistemas em seu livro: The Quantum Mechanics of Many-Body Systems. Pure and Applied Physics Series, Academic Press, 1972.

50 Quantized Hall Conductance as a Topological Invariant, Physical Review B31, p. 3372, 1985.

51 Quantum Hall Effect with Realistic Boundary Conditions, Physical Review B35, p. $2188,1987$.
} 
Christ's College [um dos Colleges da University of Cambridge/Cambridge University (UC/CU)], no qual recebeu o grau de Bacharel em Artes, em 1973. Sob a orientação do físico norte-americano Philip Warren Anderson (n.1923; PNF, 1977), Haldane defendeu a Tese de Doutorado de nome: An Extension of the Anderson Model as a Model for Mixed Valence Rare Earth Materials, em 1978, na UC/CU. Enquanto aguardava a defesa de sua Tese de Doutorado, em 1977, Haldane foi realizar estudos de pós-doutoramento no Institut Laue-Langevin (ILL), que é um Centro de Pesquisa Internacional, localizado em Grenoble, França, ficando lá até 1981, quando então foi indicado para ser Professor Assistente, na University of Southern California (USC), em Los Angeles, cargo que exerceu até 1985. Entre 1985 e 1987, foi Membro do Technical Staff, do AT \& T Bell Laboratories, em Murray Hill, New Jersey. De 1987 até 1990, foi Professor de Física, da University of California, em San Diego. Por fim, em 1990 foi escolhido Eugene Higgins Professor of Physics, da Princeton University, em Princeton, cargo que exerce até o presente momento (en.wikipedia.org/Haldane, acesso fevereiro de 2017).

Além do PNF/2016 [Topological Quantum Matter, Nobel Lecture (08/12/2016)], Haldane recebeu outras honrarias (fellows, members, medals, prizes, chair e doctor honoris causae), com destaque para: 1) Alfred P. Sloan Foundation Research Fellow, em 1984 (até 1988); 2) Member of the Advisory Board - Aspen Center for Physics, em 1990 (até 1999); 3) Fellow of the American of Arts and Sciences, em 1992; 4) Oliver E. Buckley Condensed Matter Prize (American Physical Society, em 1993); 5) Fellow of the Royal Society (FRS) (Royal Society of London, em 1996); 6) Lorentz Chair (Lorentz University/Instituut-Lorentz, em 2008); 7) Dirac Medal [Institute of Physics (IOP) (Inglaterra e Irlanda), em 2012]; e 8) Doctor Honoris Causae (Université de Cergy-Pontoise, em 2015). Observe-se que Haldane também é Distinguished Visiting Research Chair, no Perimeter Institute for Theoretical Physics, que é um Centro Internacional de Pesquisa em Física Teórica, fundado em Waterloo/Ontario, no Canadá, em 1999, pelo investidor e homem de negócios, o grego-canadense Mihal ("Mike") Lazaridis (n.1961).

Vejamos agora algumas contribuições de Haldane que o credenciaram ao nobelato e, principalmente, na Física da Matéria Condensada [p.ex.: teoria de líquidos quânticos; ondas de spin (cadeias de spin quântico); antiferromagnetismo; EHQF; FTHNN; EHQA; etc.]. Com efeito, quando ele estava na UC/CU realizando o seu Doutoramento com Anderson, eles publicaram, em 197652, o artigo: Simple Model of Multiple Charge States of Transition-Metal Impurities in Semiconductors, em que usaram o Modelo de Anderson (MA) ${ }^{53}$ sobre as impurezas magnéticas dos metais, e o estenderam aos semicondutores. É oportuno destacar que o MA, é um modelo quântico definido pelo Hamiltoniano de Anderson (HA) e usado para descrever sistemas de férmions pesados, e que contém uma ressonância estreita entre um estado

\footnotetext{
52 Physical Review B13, p. 2553, 1976.

53 Physical Review, v. 124, p. 41, 1961.
} 
de impureza magnética e um estado de condutividade elétrica. Além disso, na HA existe um potencial de troca $U$ com o átomo de impureza caracterizado por um orbital $\varphi_{\mathrm{d}}$. Há ainda nesse Hamiltoniano um termo $\mathrm{V}_{\mathrm{dk}}$, que representa o tunelamento através da barreira centrífuga da ressonância e que converte aquele orbital em um estado ressonante de energia $E_{d}$. No ILL, Haldane realizou trabalhos seminais sobre líquidos quânticos e cadeias de spin quântico, usando argumentos topológicos. Por exemplo, em 1981 (ILL: preprint SP81-95), Haldane apresentou as primeiras ideias de que o antiferromagnetismo de Heisenberg, inicialmente com spin inteiro, poderia estendido a spin fracionário (ver sua Nobel Lecture). Ainda em 198154, Haldane publicou o artigo intitulado: 'Luttinger Liquid Theory' of One-Dimensional Quantum Fluids. É oportuno registrar que, em 196355, o físico norte-americano Joaquin (“Quin”) Mazdak Luttinger (1923-1997) desenvolveu uma teoria envolvendo as interações de elétrons em metais unidimensionais, sendo que esses elétrons são ditos como estarem no Estado Luttinger-Liquido, que é um estado conhecido como líquido quântico. Registre-se ainda que a primeira ideia sobre esse tipo de "líquido" foi apresentada, em $1950^{56}$, pelo físico japonês SinItiro Tomonaga (1906-1979; PNF, 1965), daí esse efeito ser também conhecido como Líquido Tomonaga-Luttinger.

Quando Haldane estava na USC deu continuidade aos seus estudos em Física da Matéria Condensada. Assim, em 1983, publicou três trabalhos: 1) Phase Diagram of Surface Structures from Bethe Ansatz Solutions of the Quantum Sine-Gordon Model ${ }^{57}$, com os físicos dinamarqueses Tomas Bohr [filho do físico dinamarquês Aage Niels Bohr (1922-2009; PNF, 1975)] e Per Bak (1948-2002); 2) Continuum Dynamics of the 1-D Heisenberg Antiferromagnet: Identification with the O(3) Nonlinear Sigma Model (Physics Letters A93, p. 464); e 3) Nonlinear Field Theory of Large-Spin Heisenberg Antiferromagnets: Semiclassical Quantized Solitons of the One-Dimensional Easy-Axis Néel State ${ }^{58}$.

É interessante destacar que o antiferromagnetismo foi proposto pelo físico francês Louis Eugène Félix Néel (1904-2000; PNF, 1970), em 193259, ao formular um modelo de uma estrutura magnética para a qual os spins nas redes são arranjados, de um modo paralelo e antiparalelo, alternadamente, de maneira que o campo magnético resultante é nulo. Por sua vez, em 197260, os físicos, o alemão Werner Karl Heisenberg (1901-1976; PNF, 1932) e o japonês K. Yamazaki usaram os magnons [ondas de spin, descobertas pelo físico suíço Felix

\footnotetext{
54 Journal of Physics C: Solid State Physics, v. 14, p. 2585, 1981.

55 Journal of Mathematical Physics, v. 4, p. 1154, 1963.

56 Progress in Theoretical Physics, v. 5, p. 544, 1950.

57 Physical Review B28, p. 2743, 1983.

58 Review Letters, v. 50, p. 1153, 1983.

59 Annales de Physique, v. 17, p. 64, 1932.

60 Nuovo Cimento 11B, p. 125, 1972.
} 
Bloch (1905-1983; PNF, 1952), em 193061, que são estados de energia correspondente à precessão dos spins inteiros alinhados no estado fundamental] para estudar o antiferromagnetis$\mathrm{mo}^{62}$. Ainda na USC, Haldane trabalhou no EHQF. Com efeito, segundo vimos nos trabalhos nobelísticos de Thouless discutidos acima, Laughlin explicou o EHQF por intermédio da ideia de quase-partícula, usando um tipo de Hamiltoniano (HL) aplicado a sua função de onda de Laughlin: $\psi_{\mathrm{m}}$, ideia essa que foi por ele proposta, em 1983 (op. cit.). Ainda em 198363, Haldane mostrou que $\psi_{\mathrm{m}}$ representava o estado fundamental exato de uma classe de Hamiltonianos com potenciais não-locais e, com isso, encontrou um estado de fluido quântico incompressível de um gás de elétrons bidimensionais.

Na Bell Laboratories (1985-1987), Haldane deu continuidade aos trabalhos que realizara no ILL (1977-1981) e na USC (1981-1985), dentre os quais se destacam dois artigos, publicados em 1985: 1) Finite-Size Studies of the Incompressible State of the Fractionally Quantized Hall Effect and its Excitations ${ }^{64}$, com a colaboração de E. H. Rezayi e no qual diagonalizaram o HL para tratar os estados incompressíveis e suas excitações do EFHQF; 2) Quantum Dynamics and Statistics of Vortices in Two-Dimensional Superfluids ${ }^{65}$, com a participação de Yong-Shi Wu em que a dinâmica quântica bidimensional foi utilizada para investigar os vórtices de superfluidos e sua estatística.

Quando esteve na University of California, em San Diego, entre 1987 e 1990, Haldane teve oportunidade de trabalhar com Ian Affleck sobre a criticalidade das cadeias de spin quântico, resultando no artigo, publicado em 198766. Em 198867, Haldane desenvolveu um modelo para tratar cristais bidimensionais de matéria-condensada usando a TQC $(2+1)$. Neste artigo, ele obteve resultados inéditos, tais como: 1) $\mathrm{C}_{\mathrm{H}} \neq 0$, na ausência de campo magnético externo; 2) Férmions sem massa e que exibem uma "paridade anômala", daí este resultado ser conhecido como Efeito Hall Quântico Anômalo (EHQA).

Na PU, a partir de 1990, Haldane teve oportunidade de voltar a trabalhar com o HL e o EHQ, com seu colaborador Rezayi, resultando no texto publicado em 199468, em que eles estudaram a $\psi_{\mathrm{m}}$ em uma superfície cilíndrica e, ao usarem a forma polinomial dessa função

\footnotetext{
${ }^{61}$ Leipziger Vorträge: Elektronen-Interferenzen, p. 67, 1930.

62 BASSALO, J. M. F.; CARUSO, F. Heisenberg. Livraria da Física, 2014.

63 Fractional Quantization of the Hall Effect: A Hierarchy of Incompressible Quantum Fluid States. Physical Review Letters, v. 51, p. 605, 1983.
}

64 Physical Review Letters, v. 54, p. 237, 1985.

65 Physical Review Letters, v. 55, p. 2887, 1985.

66 Critical Theory of Quantum Spin Chains. Physical Review B36, p. 5291, 1987.

67 Model for a Quantum Hall Effect without Landau Levels: Condensed-Matter Realization of the "Parity Anomaly". Physical Review Letters, v. 61, p. 2015, 1988.

68 Laughlin State on Stretched and Squeezed Cylinders and Edge Excitations in the Quantum Hall Effect. Physical Review B50, p. 17199, 1994. 
para investigar as excitações limites de um fluido incompressível quântico Hall, obtiveram resultados teóricos que eram idênticos com valores numéricos conhecidos.

Concluindo esse breve relato sobre o nobelista Haldane, faremos alguns comentários sobre o EHQA que ele previu, em 1988. O mesmo foi detectado em duas ocasiões, por físicos chineses e colaboradores estrangeiros (principalmente norte-americanos) trabalhando em instituições norte-americanas. Com efeito: 1) 201369, por Cui-Zu Chang; Jinsong Zhang; Xiao Feng; Jie Shen; Zuocheng Zhang; Minghua Guo; Kang Li; Yunbo Ou; e Pang Wei usando um isolante topológico magnético ${ }^{70}$; e 2) $2014^{71}$, por Zhenhua Qiao; Wei Ren; Hua Chen; Laurent Bellaiche; Zhenyu Zhang; o norte-americano Allan H. MacDonald (n.1951) e Qian Niu, usando grafeno acoplado a um isolante antiferromagnético. Para maiores detalhes ainda sobre o EHQA, ver: Chao-Xing Liu, Shou-Cheng Zhang e Xiao-Liang Qi, The Quantum Anomalous Hall Effect: Theory and Experiment ${ }^{72}$.

\section{Kosterlitz}

Para concluir o PNF/2016, destacaremos um breve registro da vida do nobelista Kosterlitz. Ele nasceu no dia 22 de junho de 1943, em Aberdeen, na Escócia, de pais judeus alemães e emigrados para a Escócia desde 1934. Seu pai foi o renomado bioquímico alemãoescocês-inglês Hans Walther Kosterlitz (1903-1996) e sua mãe chamava-se Hannah Gresshöner. Ele realizou seus primeiros estudos no Robert Gordon's College/ Edinburgh Academy, ainda em Aberdeen, entre 1948 e 1961. Em 1962, Kosterlitz foi para o Gonville and Caius College [um dos Colleges da University of Cambridge/Cambridge University (UC/CU)], em Cambridge, Inglaterra, estudar Natural Sciences (“Ciências Naturais"), no qual obteve o Bachelor of Arts (BA), em 1965 e, posteriormente, o Master of Arts (MA), em 1966. Entre 1966 e 1969, Kosterlitz realizou seu doutorado no Brasenose College [um dos Colleges da University of Oxford (UO)]. Em 1969, na UO, Kosterlitz defendeu a Tese de Doutorado intitulada: Problems in Strong Interactions Physics.

A partir de 1969, Kosterlitz realizou os seguintes estudos de pós-doutoramento: 1) Royal Society Exchange Fellowship (Instituto di Fisica Teorica, Turim, na Itália, 1969-1970); 2) Research Fellow [Department of Mathematical Physics/Birmingham University (DMP/BirU), na Inglaterra, 1970-1973], colaborando com Thouless; e 3) Postdoctoral Fellow [Laboratory of Atomic and Solid State Physics/Cornell University (LASSP/CU), Ithaca, New York, 1973-1974]. Em 1974, Kosterlitz foi para o DMP/BU, no qual exerceu o cargo de Lecturer, até 1978, depois como Senior Lecturer, até 1980 e, por fim, como Reader, até 1981. Em 1982, Kosterlitz foi para a Brown University (BroU), em Providence, capital de Rhode Island,

\footnotetext{
69 Science, v. 340, p. 6129, 2013.

$70<$ en.wikipedia.org/Topological_Insulator>.

71 Physical Review Letters, v. 112, p. 116404, 2014.

72 Annual Review of Condensed Matter Physics, v. 7, p. 1, 2016.
} 
nos Estados Unidos, onde se encontra até o presente momento (fevereiro de 2017). Durante essa vida acadêmica, Kosterlitz foi Professor Visitante de diversas instituições de pesquisa, tais como: 1) Department of Physics/Princeton University (1978); 2) Bell Laboratories, em New Jersey (1978); 3) Department of Physics/Harvard University (1978); 4) Laboratoire de Physique/Université d'Orsay (França, janeiro-maio de 1985); 5) Saclay Nuclear Research Centre (França, junho de 1985); 6) Department of Physics/McGill University (DP/McGU) (Canadá, janeiro-março de 1991); 7) Department of Physics/Neuchatel University (Suíça, abril-maio de 1991); 8) Instituto Nacional de Pesquisas Espaciais (INPE) (São José dos Campos/São Paulo/Brasil, janeiro-fevereiro de 1998); 9) Department of Physics/BirU (março de 1998); 10) Department of Physics/Helsinki University (Finlândia, abril-junho de 1998); 11) Internacional Centre for Theoretical Physics (ICTP) (Trieste, Itália, julho de 1998); 12) DP/McGU (agosto de 1999); 13) DP/McGU (agosto de 2000); 14) Korea Institute for Advanced Study (KIAS) (Coréia do Sul, julho de 2004); e 15) KIAS (Coréia do Sul, outubrodezembro de 2005). Note-se que durante essa vida acadêmica, ele publicou $81 \operatorname{artigos}^{73}$.

Além do PNF/2016 [Topological Defects and Phase Transitions, Nobel Lecture (08/12/2016)], Kosterlitz recebeu outras honrarias (fellows, members, prizes, distinguished professor), com destaque para: 1) Maxwell Medal and Prize [Institute of Physics (IOP) (Inglaterra e Irlanda), em 1981]; Fellow (American Physical Society, desde 1993); 3) Lars Onsager Prize (American Physical Society, em 2000); e 4) Distinguished Professor (KIAS, desde 2016). Note-se que Kosterlitz é um frequente Visiting Fellow da Aalto University, na Finlândia.

A seguir, registremos algumas contribuições (principalmente nas décadas de 1970, 1980 e 1990) de Kosterlitz que o credenciaram ao nobelato, com destaque para temas relacionados com as transições de fase da matéria uni e bidimensional; vidros de spin; sistemas aleatórios; superfluidos; e dinâmica crítica. Essas contribuições começaram quando ele esteve fazendo pós-doutoramento na BirU, na década de 1970, com o também nobelista Thouless. Além desses trabalhos (registrados quando tratamos da vida de Thouless), Kosterlitz realizou outros, isoladamente e com colaboradores (inclusive: físicos brasileiros), dos quais destacaremos alguns desses trabalhos (para os demais ver seu CV). Ainda na década de 1970: 1) Critical Exponents of the Two-Dimensional XY Model ${ }^{74}$; 2) Critical Scattering for General Fields and Temperatures ${ }^{75}$, com os físicos franceses Monique Combescot e Michel Droz; 3) Bicritical and Tetracritical Points in Anisotropic Antiferromagnetic Systems ${ }^{76}$, com os físicos, o norte-americano David Robert Nelson (n.1951) e o inglês-norte-americano Michael Ellis

\footnotetext{
73 Michael Kosterlitz, Curriculum Vitae. Disponível em: <en.wikipedia.org>. Acesso em: fev. 2017.

74 Journal of Physics C: Solid State Physics, v. 7, p. 1046, 1974.

75 Physical Review Letters, v. 33, p. 705 (1974)]

76 Physical Review B13, p. 412 (1976)]
} 
Fisher (n.1931); 4) Phase Transitions in Long-Range Ferromagnetic Chains ${ }^{77}$; 5) The dDimensional Coulomb Gas and the Roughening Transition ${ }^{78}$; 6) Universal Jump in the Superfluid Density of Two-Dimensional Superfluids ${ }^{79}$, com Nelson; 7) Phase Transitions in Layered Magnetic Systems ${ }^{80}$, com M. A. Santos; 8) Real Space Renormalization Group for Critical Dynamics ${ }^{81}$, com Ya'akov Achiam.

Nas décadas de 1980, 1990 e 2000, Kosterlitz publicou artigos com colaboradores, com destaque para os realizados com o físico brasileiro Enzo Granato (n.1955), que defendeu seu doutorado com ele na Brown University, em 1986: 1) Non-Uniform Long-Range Order in Certain Random Systems ${ }^{82}$, com Robert A. Pelcovits; 2) Commensurate-Incommensurate Transitions and a Floating Devil's Staircase ${ }^{83}$, com Benjamin Horowitz, T. Bohr e Hans-Jörg Schulz; 3) A Random Anisotropy Model: 1/N Expansion for Gaussian Fluctuations in the Spin Glass Phase and the Replica Symmetry Breaking Instability ${ }^{84}$, com A. Khurana e A. Jagannathan; 4) Scaling of Conductivities in the Fractional Quantum Hall Effect ${ }^{85}$, com Laughlin, Marvin L. Cohen (n.1935) (canadense-norte-americano), Herbert Levine, Stephen B. Libby e Adrianus M. M. Pruisken; 5) Critical Behaviour of Coupled XY Models ${ }^{86}$, com Granato e S. Poulter; 6) Frustrated XY Model with Unequal Ferromagnetic and Antiferromagnetic Bonds $^{87}$, com Granato; 7) Quenched Disorder in Josephson-Junction Arrays in a Transverse Magnetic Field 88 , com Granato; 8) Resistance Oscillations in a Josephson-Junction Array in a Magnetic Field ${ }^{89}$, com Granato; 9) Renormalisation Group Treatment of the Long Range One Dimensional Ising Model with Random Fields ${ }^{90}$, com P. O. Weir; 10) Equilibrium Study of Strained Epitaxial Layers on a Rigid Substrate ${ }^{91}$, com Granato e See-Chen Ying; 11) Helicity

\footnotetext{
${ }^{77}$ Physical Review Letters, v. 37, p. 1577, 1976.

78 Journal of Physics C: Solid State Physics, v. 10, p. 3753, 1977.

${ }^{79}$ Physical Review Letters, v. 39, p. 1201, 1977.

${ }^{80}$ Journal of Physics C: Solid State Physics, v. 11, p. 2835, 1978.

${ }^{81}$ Physical Review Letters, v. 41, p. 128, 1978.

82 Journal of Physics A: Mathematical, Nuclear and General, v. 16, p. L763, 1983.

${ }^{83}$ Physical Review B28, p. 6596, 1983.

${ }^{84}$ Nuclear Physics B240, p. 1, 1984.

85 Physical Review B32, p. 1311, 1985.

86 Physical Review B33, p. 4767, 1986.

${ }^{87}$ Journal of Physics C: Solid State Physics, v. 19, p. L59, 1986.

88 Physical Review B33, p. 6533, 1986.

${ }^{89}$ Physical Review B34, p. 2026, 1986.

${ }^{90}$ Physical Review B36, p. 5760, 1987.

91 LATIN AMERICAN CONFERENCE EON SEMICONDUCTOR PHYSICS, 1987, São Carlos, Brasil. Proceedings...
} 
Modulus of a Frustrated XY Model ${ }^{92}$, com Granato e Ying; 12) Disorder in Josephson Junction Arrays ${ }^{93}$; com Granato; 13) Equilibrium Theory of Strained Epitaxial Layers ${ }^{94}$, com Granato e Ying; 14) Melting of a Free Bilayer ${ }^{95}$, com Granato e Ying; 14) Disorder in Josephson Junction Arrays in a Magnetic Field ${ }^{96}$, com Granato; 15) Superconductor-Insulator Transition and Universal Resistance in Josephson Junction Arrays in a Magnetic Field ${ }^{97}$, com Granato; 16) Phase Transitions in a Coupled XY-Ising Model ${ }^{98}$, com Granato, Jooyoung Lee e Peter M. Nightingale; 17) Phase Transitions in Fully Frustrated XY Models ${ }^{99}$, com Granato e Lee; 18) Fully Frustrated Junction Arrays and Coupled XY-Ising Models ${ }^{100}$, com Granato e Lee; 19) Numerical Studies of Phase Transitions ${ }^{101}$, com Lee e Granato; 20) Numerical Studies of the Two-Dimensional XYModel with Symmetry Breaking Fields ${ }^{102}$, com Tapio. AlaNissila, Granato, Kari Kankaala e Ying; 21) Conformal Anomaly and Critical Exponents of a Coupled XY- Ising Model ${ }^{103}$, com Nightingale e Granato; 22) Critical Behavior of Josephson Junction Arrays at $\mathrm{f}=1 / 2^{104}$, com Granato e Nightingale; 23) Edge Effects in a Frustrated Josephson Junction Array with Modulated Couplings ${ }^{105}$, com Granato e Mikhail V. Simkin; 24) Surface Instability and Dislocation Nucleation in Strained Epitaxial Layers ${ }^{106}$, O. S. Trushin, Granato, Ying, Alla-Nissila e P. T. Salo; e 25) Pattern Selection in a Phase Field Model for Directional Solidification ${ }^{107}$, com Granato e o também físico brasileiro Raimundo Nogueira da Costa Filho n.1969) (a quem, nesta oportunidade, agradeço a leitura crítica deste texto sobre o PNF/2016), que realizou pós-doutoramento com Kosterlitz, na Brown University, em 2001.

\footnotetext{
92 Journal of Applied Physics, v. 64, p. 5636, 1988.

93 Physica B152, p. 62, 1988.

${ }^{94}$ Physical Review B39, p. 3185, 1989.

95 Physical Review B39, p. 4444, 1989.

${ }^{96}$ Physical Review Letters, v. 62, p. 823, 1989.

${ }^{97}$ Physical Review Letters, v. 65, p. 1267, 1990.

98 Physical Review Letters, v. 66, p. 109, 1991.

${ }^{99}$ Physical Review B43, p. 11531, 1991.

100 Physical Review B44, p. 4819, 1991.

${ }^{101}$ LANDAU, D. P.; MON, K. K.; SChUTtLer, H. B. (Ed). Computer Simulations in Condensed Matter Physics IV, Heidelberg: Springer, 1992. p. 28.

102 Physical Review B50, p. 12692, 1994.

103 Physical Review B52, p. 7402, 1995.

104 Physica B222, p. 266, 1996.

105 Physical Review B57, p. 3602, 1998.

106 Brazilian Journal of Physics, v. 32, p. 369, 2002.

107 Physica A: Statistical Mechanics and Applications, v. 354, p. 333, 2005.
} 